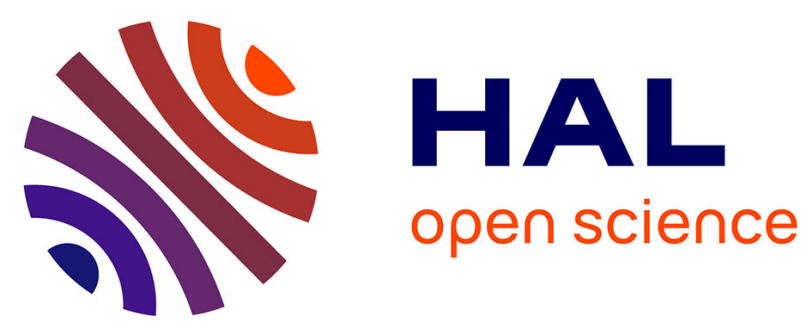

\title{
Importance of Phase Changes in Titan's Lower Atmosphere. Tools for the Study of Nucleation
}

\author{
Lionel Guez, Paul Bruston, François Raulin, Christian Régnaut
}

\section{To cite this version:}

Lionel Guez, Paul Bruston, François Raulin, Christian Régnaut. Importance of Phase Changes in Titan's Lower Atmosphere. Tools for the Study of Nucleation. Planetary and Space Science, 1997, 45, Issue 6, p. 611-625. 10.1016/S0032-0633(97)00018-4 . hal-00013560

\section{HAL Id: hal-00013560 https://hal.science/hal-00013560}

Submitted on 9 Nov 2005

HAL is a multi-disciplinary open access archive for the deposit and dissemination of scientific research documents, whether they are published or not. The documents may come from teaching and research institutions in France or abroad, or from public or private research centers.
L'archive ouverte pluridisciplinaire HAL, est destinée au dépôt et à la diffusion de documents scientifiques de niveau recherche, publiés ou non, émanant des établissements d'enseignement et de recherche français ou étrangers, des laboratoires publics ou privés. 


\title{
IMPORTANCE OF PHASE CHANGES IN TITAN'S LOWER ATMOSPHERE. TOOLS FOR THE STUDY OF NUCLEATION
}

\author{
L. Guez ${ }^{1}$, P. Bruston ${ }^{1}$, F. Raulin ${ }^{1}$ and C. Régnaut ${ }^{2}$
}

${ }^{1}$ Laboratoire Interuniversitaire des Systèmes Atmosphériques

Universities Paris 7 and Paris 12, CNRS - U.R.A. 1404

61, avenue du général de Gaulle

94010 Créteil cedex

France

2Laboratoire de Physique des Milieux Désordonnés

University Paris 12

61, avenue du général de Gaulle

94010 Créteil cedex

France

Submitted to Planetary and Space Science, April 1, 1996

Revised November 23, 1996

Final version February 21, 1997

Correspondence to: L. Guez 


\section{Abstract}

The uncertainty about possible supersaturation of methane, condensation of volatile species and the existence of clouds in Titan's lower atmosphere affects our understanding of photochemistry, the nature of the surface and the atmospheric thermal structure. Indeed, photochemistry depends on the depth of penetration of energetic photons, affected by methane abundance. Radar and infrared observations of bright surface regions have been explained by rain washing of highlands. As for the thermal profile, it is sensitive to $\mathrm{CH}_{4}-\mathrm{N}_{2}$ gas opacity, cloud opacity and could be influenced by latent heat exchange. A rudimentary model with no methane supersaturation and gas transport by eddy diffusion indicates a methane latent heat release of $0.2 \mathrm{~W} \mathrm{~m}^{-2}$ between 20 and $30 \mathrm{~km}$ altitude for a surface mole fraction of $4.4 \%$ and an eddy diffusion coefficient of $0.2 \mathrm{~m}^{2} \mathrm{~s}^{-1}$. Description of nucleation seems to be one of the first improvements which should be included in a model of phase changes. The suspicion of difficult methane nucleation comes from analysis of Voyager IRIS spectra. Moreover, species are expected to condense to the solid phase, which excludes very efficient nucleation and condensation processes associated with the presence of a liquid phase, such as deliquescence. The classical theory of heterogeneous nucleation, despite its deficiencies, is employed in atmospheric models, owing to its general nature and relative simplicity. Yet, it requires physical quantities for which experimental values do not exist. We show how surface free enthalpies of solids and contact angles may be linked to other material properties which are within reach of laboratory experiments, mainly ultraviolet absorption spectra of solid phases. We find that a value of $10^{-9} \mathrm{~s}^{-1}$ $10^{-7} \mathrm{~s}^{-1}$ for the 'critical nucleation rate' (per nucleus) is adapted to the case of Titan, though we question the ability of the critical rate concept to make predictions for the condensation altitudes. A possible consequence of difficult methane nucleation is periodic evolution of the lower atmosphere, on a time scale of the order of $10^{2}$ years. 


\section{Introduction}

Titan, Saturn's largest satellite, has been an important subject of study since the plethora of observations brought by the Voyager 1 and 2 missions, in 1980 and 1981. Titan has a dense atmosphere, mainly composed of $\mathrm{N}_{2}$, with a few percent $\mathrm{CH}_{4}$ and a rich array of trace organic compounds, making Titan a planetary object of interest for exobiology (Raulin et al., 1994). The aerosols observed in Titan's atmosphere are thought to be synthesized photochemically at high altitudes (> $300 \mathrm{~km}$ ) (see Chassefière and Cabane, 1995). Laboratory simulations of Titan's atmosphere yield such high-molecular weight solid products, termed "tholins" (e. g. Coll et al., 1997). The aerosols fall to the surface and, as they arrive in the colder lower part of the atmosphere below about $100 \mathrm{~km}$, they may serve as condensation nuclei for low-molecular weight species (Sagan and Thompson, 1984).

The aerosol distribution above the condensation region was modeled by McKay et al. (1989), and by Cabane et al. (1993), who took into account the probable aggregate-like structure of those aerosols. Frère (1989) proposed a model of the aerosol distribution down to the ground (partly in Frère et al. (1990) too). It described in a simple way condensation of light organic species (but not nucleation) in the lower stratosphere assuming just saturated mole fractions in the gas phase for all considered species. Frère (1989) followed Sagan and Thompson (1984) and supposed that the condensation of a species (other than methane) begins where the saturation-mole fraction reaches the estimated stratospheric mole fraction (a mean value which is independent of altitude). However, because of the downward flux, the mole fraction decreases with altitude and is probably lower where condensation begins than its mean observed value. Therefore, the altitudes of condensation in Sagan and Thompson (1984) and Frère (1989) are probably too high. Condensation was also taken into account by chemical models (Yung et al., 1984; Lara et al., 1994; Toublanc et al., 1995), although those models only needed to compute the gas loss due to condensation and did not follow the aerosol distribution.

In the present work, we first review the influence of phase changes and aerosols in the lower atmosphere on other properties of Titan's atmosphere and surface. Then we address the pertinence of including the description of nucleation in a model of aerosols of the lower atmosphere. We try to open paths for the evaluation of the quantities required to compute nucleation rates, linking those quantities to material properties which are within reach of laboratory experiments. Finally, we look for an adequate definition of the 'critical nucleation rate' on Titan. This concept allows a first glimpse of possible consequences of nucleation difficulties in Titan's atmosphere.

\section{Importance of aerosols in Titan's lower atmosphere}

In this section, we attempt to point out how our knowledge of some properties of Titan depends on information on the size distribution of the aerosols in the 
lower atmosphere, or their chemical structure, or phase changes in the lower atmosphere. In particular, we envisage that volatile organic species might supersaturate or even that their condensation might be completely inhibited (see $\S 3$ ).

\subsection{Radiation and heat transfer in the lower atmosphere}

In order to be properly interpreted, several properties of Titan require taking into account the possible presence of clouds in the lower atmosphere and the effect of condensation on the amount of gaseous methane. Such is the case with brightness temperature spectra in the 200 to $600 \mathrm{~cm}^{-1}$ wave number range (an infrared "window"), observed by the IRIS instrument on Voyager, the geometric albedo in the visible at wavelengths greater than $0.6 \mu \mathrm{m}$ and in the near infrared (Neff et al., 1984; Fink and Larson, 1979; Griffith, 1993; Lemmon et al., 1995; Cousténis et al., 1995; Smith et al., 1996) and the temperature profile of the lower atmosphere, deduced from the Voyager radio-occultation experiment. Therefore, workers who analysed IRIS infrared "window" spectra (most recently Toon et al., 1988; McKay et al., 1989; Courtin et al., 1995) or the geometric albedo (McKay et al., 1989; Griffith et al., 1991; Toon et al., 1992), or the temperature profile (McKay et al., 1989) needed to propose answers to the questions: What is the opacity of clouds at each wavelength studied? What is the vertical distribution of cloud opacity? What is the abundance of gaseous methane as a function of altitude? Toon et al. (1988) and McKay et al. (1989) assumed that the abundance of methane is limited by saturation in the troposphere and that cloud extinction (if any) is spatially distributed proportionally to methane abundance in the saturation region. Then they treated cloud opacity at a reference wavelength and cloud particle size as free parameters to be constrained by observations. Courtin et al. (1995) had a similar approach. Although their hypotheses were less restrictive - as they considered the possibility of methane supersaturation and that of a cloud concentrated near the tropopause -, cloud opacity and particle size, and maximum supersaturation were still free parameters. The best fit was obtained with significant methane supersaturation and no cloud opacity but a model with no supersaturation and a cloud concentrated at the tropopause was also acceptable. Thus, it is an important issue to examine directly, from physical modeling of aerosols and phase changes, the validity of hypotheses on methane abundance and distribution of cloud opacity. In particular, if probable methane supersaturation emerged from such modeling, it might have profound implication on the way we understand the temperature profile, since the $\mathrm{CH}_{4}-\mathrm{N}_{2}$ pressure-induced absorption is a major contributor to the opacity in the 200 $\mathrm{cm}^{-1}-600 \mathrm{~cm}^{-1}$ infrared window of the atmosphere (McKay et al., 1989; McKay et al., 1991).

Phase changes also influence the thermal profile in the lower atmosphere through the exchange of latent heat. As a preliminary assessment of the importance of latent heat release in Titan's troposphere, we now propose a rudimentary model of methane condensation. The model is one-dimensional and 
the basic assumptions are: gas is transported through eddy diffusion, not convection (although convection may exist too (Awal and Lunine, 1994)); no supersaturation; no evaporation of methane rain. Those hypotheses allow a simple analytic treatment. To begin with, we choose for the mole fraction of methane at the surface: $\mathrm{x}(\mathrm{z}=0)=4.4 \%$ (about $36 \%$ relative humidity), and for the eddy diffusion coefficient: $K=0.2 \mathrm{~m}^{2} \mathrm{~s}^{-1}$. The methane gas flux density $\Phi$ is related to the methane mole fraction $x$ by:

$\Phi=-K N \frac{d x}{d z}$

where $N$ is the total number density of gas molecules. We use the background atmospheric profiles given by Lellouch (1990) and the methane solid-gas equilibrium pressure from Kirk and Ziegler (1965).

Between the surface and some intersection altitude $\mathrm{z}_{i}, x$ is lower than its saturated value, $x_{s}$. From $z_{i}$ up almost to the cold trap (at about $30 \mathrm{~km}$ ), $x$ equals $x_{\mathrm{S}}$. We suppose that the chemical sources and sinks of methane are negligible in the troposphere, then any variation of $\Phi$ can only be due to phase change. As no evaporation is considered, $\Phi$ is constant between the surface and $\mathrm{z}_{\mathrm{i}}$. Therefore, using equation (1), the flux near the surface $\Phi_{\text {surf }}$ may be regarded as a function of the intersection altitude:

$\Phi_{\operatorname{surf}}\left(z_{i}\right)=\frac{x(0)-x_{s}\left(z_{i}\right)}{\int_{0}^{z_{i}} \overline{d z}}$

$\Phi_{\text {surf }}\left(z_{i}\right)$ is plotted in figure 1 . Above $z_{i}, \Phi$ equals the saturation-flux $\Phi_{\mathrm{s}}$ : $\Phi_{S}=-K N \frac{d x_{s}}{d z}$

$\Phi_{\mathrm{s}}$ decreases with increasing altitude (see figure 1), so methane condenses between $\mathrm{Z}_{i}$ and the cold trap.

Let us show that the gas flux $\Phi$ can undergo no discontinuity at $\mathrm{z}_{\mathrm{i}}$. We first note that, irrespective of hypotheses on gas transport, supersaturation and evaporation, a discontinuous gas flux means an infinite evaporation or condensation rate. From the point of view of aerosols bearing condensed methane, a continuous gas flux means that their number density does not change instantaneously (by a non-infinitesimal value) when they cross $\mathrm{z}_{\mathrm{i}}$, neither do their radii. With our hypotheses (negligible supersaturation and evaporation, transport by eddy diffusion with a continuous $K$ profile), we have been able to define the altitude $\mathrm{z}_{i}$ and the flux profile $\Phi_{\mathrm{s}}$, and the gas flux $\Phi$ may only decrease with increasing altitude (no evaporation):

$\Phi_{\text {surf }} \geq \Phi_{s}\left(z_{i}\right)$

Moreover, $x$ is lower than $\mathrm{x}_{\mathrm{S}}$ at altitudes below $\mathrm{z}_{\mathrm{i}}$ so:

$\frac{d x}{d z}\left(z \longrightarrow z_{i}\right) \geq \frac{d x_{s}}{d z}\left(z \underset{<}{<} z_{i}\right)$

$\Rightarrow \Phi_{\text {surf }} \leq \Phi_{s}\left(z_{i}\right)$

Hence, the gas flux must be continuous at $\mathrm{z}_{\mathrm{i}}$. We call $\mathrm{z}_{\mathrm{i}}{ }^{*}$ that particular value of $\mathrm{z}_{\mathrm{i}}$ which gives continuous flux. Solving the equation: 
$\Phi_{s}\left(z_{i}{ }^{*}\right)=\Phi_{\operatorname{surf}}\left(z_{i}{ }^{*}\right)$

We obtain (see figure 1):

$\mathrm{z}_{\mathrm{i}}^{*} \approx 19 \mathrm{~km}$

$\mathrm{x}_{\mathrm{S}}\left(\mathrm{z}_{\mathrm{i}}^{*}\right) \approx 2.5 \%$

$\Phi_{\text {surf }}\left(\mathrm{z}_{\mathrm{i}}^{*}\right)=\Phi(\mathrm{z}=0) \approx 1.5 \times 10^{19} \mathrm{~m}^{-2} \mathrm{~s}^{-1}$

This corresponds to the evaporation of about $3 \mathrm{~cm}$ of liquid methane per (terrestrial) year. On the other hand, the gas flux that escapes into the stratosphere through the cold trap is the chemical destruction flux: $1.3 \times 10^{14}$ $\mathrm{m}^{-2} \mathrm{~s}^{-1}$ (Toublanc et al., 1995) ${ }^{1}$. It is only a minute fraction of the methane flux that cycles in the troposphere. Consequently, the column condensation rate of methane is approximately the surface flux and the latent heat released in the troposphere from methane condensation, per unit area, is:

$j_{q}=\Phi(z=0) \frac{L}{N_{A}}$

where $L$ is the molar latent heat of vaporization or sublimation and $\mathrm{N}_{\mathrm{A}}$ is the Avogadro number. From the solid-gas and liquid-gas equilibrium pressures (Kirk and Ziegler, 1965), the latent heats of vaporization and sublimation near the triple point are about $8500 \mathrm{~J} \mathrm{~mol}^{-1}$ and $9700 \mathrm{~J} \mathrm{~mol}^{-1}$ respectively. For the precision needed here, we may use $L=9 \mathrm{~kJ} \mathrm{~mol}^{-1}$, so:

$\mathrm{j}_{\mathrm{q}} \approx 0.2 \mathrm{~W} \mathrm{~m}^{-2}$

That is about $5 \%$ of the total energy radiated by Titan's surface:

$\mathrm{M}=\sigma_{\mathrm{S}} \mathrm{T}^{4}(\mathrm{z}=0) \approx 4.4 \mathrm{~W} \mathrm{~m}^{-2}$

where $\sigma_{S}$ is the Stefan-Boltzmann constant. For comparison, on Earth, the global mean annual evaporation of water (equal to the global mean annual precipitation) amounts to about $1 \mathrm{~m}$ (Pruppacher and Klett, 1978, page 349) so that:

$\mathrm{j}_{\mathrm{q}, \text { Earth }} \approx 80 \mathrm{~W} \mathrm{~m}^{-2}$

or about $20 \%$ of $\mathrm{M}_{\mathrm{Earth}}$ (Liou, 1992, figure 1.6) (note that the latent heat of vaporization is about 5 times greater for water than for methane).

The surface flux of methane increases with its mole fraction at the surface. Thus, an upper limit is obtained from the surface flux at $100 \%$ relative humidity: $\Phi(\mathrm{z}=0) \leq \Phi_{\mathrm{s}}(0) \approx 2 \times 10^{20} \mathrm{~m}^{-2} \mathrm{~s}^{-1}$

corresponding to the evaporation of about $50 \mathrm{~cm}$ of liquid methane per (terrestrial) year and a release of latent heat reaching about $70 \%$ of the surface thermal emission $M$. The surface flux also varies with the eddy diffusion coefficient. If $K$ is uniform in the troposphere then $\Phi_{\text {surf }}\left(z_{i}\right)$ is proportional to $K$

${ }^{1}$ The reader might notice that the model assumptions as well as the values used for $\mathrm{x}(\mathrm{z}=0)$ and $K$ correspond to those in Toublanc et al. (1995). The value quoted by Toublanc et al. for the methane gas flux at the surface $\left(7 \times 10^{14} \mathrm{~m}^{-2} \mathrm{~s}^{-1}\right)$ is orders of magnitude lower than the value obtained here. There is a mistake in their computation, acknowledged by $\mathrm{D}$. Toublanc (personal communication). 
(equation (2)), as is $\Phi_{\mathrm{s}}$. So the solution to equation (3) is not affected by a change of $K$. Hence, when $K$ changes, the abundance profile does not change, but the flux at each altitude varies proportionally to $K$. In particular, the surface flux scales proportionally to $K$.

Toon et al. (1992) and Toublanc et al. (1995) propose profiles of the eddy diffusion coefficient down to the ground. These authors are able to constrain the value of $K$ in the lower stratosphere by fitting the abundance of minor components deduced from IRIS observations and the stratospheric profile of HCN abundance deduced from millimeter observations. Toon et al. (1992) also use the geometric albedo measured in the near ultraviolet and near infrared. The derived stratospheric $K$ values are just extended to the troposphere. Toon et al. (1992) comment that the tropospheric $K$ value is little constrained because of other uncertainties in their model. Incidentally, we note that they only take into account the influence of $K$ on the distribution of haze aerosols (without condensed volatile species), while methane cloud opacity is a free parameter of their model. In fact, as can be seen from the model presented here, the amount of condensed methane should depend on $K$ so there is a potential constraint on tropospheric $K$ from the geometric albedo in the visible (at wavelengths greater than $0.6 \mu \mathrm{m}$ ) and near infrared (see Toon et al., 1992). In the end, the only available constraint on the tropospheric value of $K$ comes from Flasar et al. (1981). From the observed latitudinal distribution of brightness temperature at $530 \mathrm{~cm}^{-1}$ and assumptions on the general circulation, they infer an order-ofmagnitude upper limit:

$\mathrm{K} \leqslant 0.1 \mathrm{~m}^{2} \mathrm{~s}^{-1}$

This agrees with the values from Toon et al. (1992) $\left(0.5 \mathrm{~m}^{2} \mathrm{~s}^{-1}\right)$ and Toublanc et al. (1995) $\left(0.2 \mathrm{~m}^{2} \mathrm{~s}^{-1}\right)$. So the values quoted above for the surface flux and the latent heat release should give a maximum order of magnitude.

Moreover, we note that methane rain evaporation would permit a lower surface flux. The corresponding $x$ profile would be more rounded between 0 and $z_{i}$ (as the flux would increase with altitude $z$ ), and a lower lapse rate $\left(\left|\frac{d x}{d z}\right|\right)$ at the surface would be sufficient to join tangentially the $\mathrm{x}_{\mathrm{s}}$ profile. Supersaturation would also tend to diminish the surface flux, allowing $\Phi$ to be lower than $\Phi_{\mathrm{S}}$ at $\mathrm{z}_{\mathrm{i}}$.

Latent heat exchange is not included in the model of McKay et al. (1989). The above simple condensation model shows a possible noteworthy release of latent heat in the $[20 \mathrm{~km}, 30 \mathrm{~km}]$ altitude range. If methane does indeed condense in the upper troposphere, there should also be evaporation and absorption of latent heat under $20 \mathrm{~km}$ (Lorenz, 1993a). Interestingly, the modeled temperature profile (McKay et al., 1989) seems to be stubbornly colder than the radio-occultation derived profile between $20 \mathrm{~km}$ and $30 \mathrm{~km}$. If tropospheric methane condensation really occurs then taking latent heat exchange into account might be an answer to this discrepancy. 


\subsection{Properties of Titan's surface}

Ground-based radar and infrared observations (cf. Lunine, 1993; Griffith, 1993; Lemmon et al., 1995; Cousténis et al., 1995) and Hubble Space Telescope imaging (Smith et al., 1996) indicate a heterogeneous surface. Some ideas about possible surface types are useful to interpret the surface albedo spectra that may be derived from those observations (see speculations by Lorenz, 1993b).

Though a global ocean is ruled out by Titan's surface heterogeneity, the presence of lakes or seas remains plausible (e. g. Lorenz, 1994). As suggested by Lunine (1993), the optical properties of those liquid areas could be altered by the presence of solid particles maintained by stirring in the surface layer. Apart from stirring of the surface layer, another possible cause of the presence of dust on liquid areas is that falling particles are kept afloat by surface tension forces. That may occur if those particles are bare tholins, but not if the particles are tholins surrounded by condensed low-molecular-weight hydrocarbons, with an outer methane or ethane shell. Indeed, even if the outer methane or ethane shell is solid then the surface tension forces exerted on the shell by a methaneethane-nitrogen(-argon) liquid (before dissolution of the shell) are likely to be negligible. Lorenz (1993a) finds that even if methane condenses on aerosols, it must later evaporate before reaching lowland terrain. It is also interesting to investigate the possible presence and evaporation of other volatile layers on aerosols to estimate the buoyancy of the aerosols that finally reach the surface.

Besides, radar reflectivity (Muhleman et al., 1992; Muhleman et al., 1995) and spectral shape of the near infrared albedo (Cousténis et al., 1995) of some regions of the surface ("bright" regions) suggest water ice from the exposed bedrock rather than a deposit of photochemical solid organic products or than methane-ethane lakes. Washing of highland terrain by methane rainfall has been suggested to explain exposing of bedrock (Griffith et al., 1991; Lunine, 1993; Lorenz, 1993a; Smith et al., 1996). However, methane condensation may be inhibited in the troposphere. In that case, there is probably no ethane or propane precipitation in the lower troposphere either because if there were, it should be liquid in the last few kilometers above the surface (considering the temperatures in the lower troposphere), and then it would very efficiently induce methane condensation. Admittedly, there should still be a steady condensation of ethane and propane on contact with Titan's surface (or in its immediate vicinity), since these compounds are continuously produced in the stratosphere and must have a sink somewhere. Yet, liquid ethane and propane trickling alone from highland terrain might be less efficient for surface washing than methane rain, due to lower mass flux and the absence of raindrop impacts. Again, physical modeling of phase changes in the atmosphere is warranted.

\subsection{Chemistry in the gas phase}

For most products of atmospheric chemistry (species for which there is a global net chemical production in the gas phase), condensation in the lower 
stratosphere is potentially a major sink of gas molecules, compared to condensation on the surface or atmospheric escape (e. g. Yung et al., 1984). As for methane, its abundance in the stratosphere may or may not be limited by saturation at the cold trap, depending on the efficiency of methane condensation. The sensitivity of photochemistry in the stratosphere and above to the efficiency of condensation in the lower atmosphere is not well known. To appreciate this, we briefly review how chemical models have taken the condensation sink into account and how the sensitivity has been tested.

Yung et al. (1984) impose a downward velocity of each gas at the tropopause, which implicitly supposes condensation in the troposphere or on the surface. They add stratospheric loss, using the following condensation rate (number of molecules condensing on aerosols per unit volume of atmosphere and per unit time) for a given species:

$C=\left(10^{-9} s^{-1}\right) \frac{p-p_{s}}{k_{B} T}$

where $p$ is the partial pressure of the species, $p_{s}$ is its saturation pressure, $\mathrm{k}_{\mathrm{B}}$ is the Boltzmann constant and $T$ is the temperature. That amounts to making a hypothesis on the aerosol number density $\tilde{N}$ and mean radius $r$ in the stratosphere, since the expression giving the condensation rate per unit volume on spherical aerosols is (neglecting the Kelvin effect) (e. g. Seinfeld, 1986, page 336):

$C=(4 \pi r D \tilde{N} f) \frac{p-p_{s}}{k_{B} T}$

where $D$ is the Brownian diffusion coefficient of the species (in the gas phase) and $f$ is a function of the Knudsen number $\mathrm{N}_{\mathrm{Kn}}$ (mean free path of gas molecules of the condensing species divided by $r$ ) close to 1 when $\mathrm{N}_{\mathrm{Kn}}$ is small

("continuous" regime).

Yung et al. (1984) seem to find that for all species, $p$ remains close to $p_{s}$ in the condensation region: the eddy diffusion coefficient $K$ is low enough and $r$ and $\tilde{\mathrm{N}}$ are high enough to allow the condensation of all the excess mass of incoming condensable species.

Romani et al. (1993), for their chemical model of Neptune's atmosphere, compute the condensation rate with an equation similar to equation (6). They use a constant radius, which they constrain from observations. Then they assume that there is a separate distribution of aerosols for each condensing species (each species condenses only on its own crystals), which permits them (using again the chosen radius) to relate $\tilde{\mathrm{N}}$ to the integrated net chemical production rate.

Lara et al. (1994) also include the condensation loss term given by equation (6), with Cabane et al. (1992)'s aerosol distribution as an input, although Cabane $e t$ al.'s model does not incorporate condensation. 
In the model of Toublanc et al. (1995), there is no such parameterization. The partial pressures are simply not allowed to exceed their saturated values. The excess mass is lost to the condensed phase.

Condensation can directly affect chemistry when both processes take place in the same region (e. $g$. for diacetylene $\mathrm{C}_{4} \mathrm{H}_{2}$ on Uranus, see Summers and Strobel, 1989). On Titan, the contribution of chemistry under about $100 \mathrm{~km}$ to the column integrated reaction rates seems to be negligible, at least for major reactions destroying and producing methane and $\mathrm{C}_{2}$ hydrocarbons (Yung et al., 1984). Even when the regions of condensation and chemistry are distinct, varying the efficiency of condensation in the lower stratosphere and in the troposphere must in principle affect chemistry at higher altitudes through gas diffusion. Quantitatively, however, the particular way of taking condensation into account in a chemical model has uncertain importance for strictly chemical results. (By 'strictly chemical' results, we mean the profiles of mole fraction in the gas phase above the condensation region, and the integrated production or destruction rates.) Yung et al. (1984) and Romani et al. (1993) find that the amounts condensed are insensitive to moderate changes in their condensation parameters. So not only are the chemical production rates unaffected but also the partition between the two modes of removal (condensation and gas diffusion through the lower boundary), because the eddy diffusion coefficient is low enough, and $r$ and $\tilde{\mathrm{N}}$ are high enough that condensation remains the dominant sink. More drastic perturbations of condensation, due for instance to nucleation delays, might have higher influence on atmospheric chemistry. In particular, if methane condensation is inhibited, its abundance may be significantly increased in the stratosphere and above, raising the altitude of optical depth unity at Lyman $\alpha$. This changes the altitudes where photochemistry takes place, hence the ambient temperatures and pressures for photochemistry. (Such an influence of methane abundance at the cold trap is described by Romani et al. (1993), though not in relation with the efficiency of methane condensation [the lower boundary of their model is the tropopause], but due to re-estimation of the stratospheric mixing ratio after the Voyager encounter with Neptune.)

\section{Need for nucleation modeling on Titan}

Difficult nucleation in the cold atmospheres of the outer solar system has been suggested by Moses et al. (1992). There are two qualitative reasons why it seems to be worth considering the process of nucleation in Titan's atmosphere, not just assuming nucleation to be instantaneous for all species ( $i$. $e$. very efficient as soon as negligible supersaturation is achieved).

First, the analysis of IRIS spectra in the $200 \mathrm{~cm}^{-1}-600 \mathrm{~cm}^{-1}$ wave number range by Courtin et al. (1995) shows that those are best fit with no cloud opacity but with significant supersaturation of methane (of the order of 100\%) in the troposphere. This suggests difficult methane nucleation. We may not conclude that a real signature of difficult nucleation is found because an acceptable fit to the brightness spectra is also obtained with no supersaturation and a cloud very 
near the tropopause. Furthermore, as noted by Courtin et al. (1995), supersaturation and the absence of cloud opacity could be explained not by difficult nucleation but simply by the dynamics of condensation (after nucleation), gas transport and aerosol transport. For example, assuming methane condenses on photochemical aerosols (possibly covered with volatile organic species), the incoming flux of those aerosols might be small so that condensation is slow compared to re-supplying of gas by eddy diffusion, and the number density of methane crystals or droplets might be too small to create a uniform optically thick deck of clouds. There still remains a suspicion of difficult nucleation and an encouragement to model the physics of nucleation.

Second, we are led by the study of Earth's atmosphere which guides us on conditions which are favorable or not to nucleation. In the terrestrial troposphere, nucleation is quite efficient and supersaturation of water vapor (with respect to liquid-vapor equilibrium) rarely exceeds a few percent. Indeed, most often, liquid water drops form through a very effective process: heterogeneous nucleation on solid aerosols which are soluble, or partially soluble, in water (Pruppacher and Klett, 1978, pages 162, 225, 237). Nucleation is then the deliquescence of the solid soluble part. With regard to ice particles, they start principally from the supercooled liquid phase rather than directly from the vapor (Keesee, 1989). Thus, nucleation in the terrestrial troposphere benefits from two combined favorable conditions: the nucleation of the liquid rather than solid phase (either because the temperature is above $0{ }^{\circ} \mathrm{C}$ or because the supercooled liquid phase is possible) and the solubility of condensation nuclei.

Such conditions may not be present on Titan. For almost all species liable to condense in Titan's lower stratosphere, the triple point temperature is greater than the temperature in the expected region of condensation (Sagan and Thompson, 1984; Frère, 1989). Propane $\left(\mathrm{C}_{3} \mathrm{H}_{8}\right)$, 3-methyl-hexane $\left(\mathrm{C}_{2} \mathrm{H}_{5} \mathrm{CH}\left(\mathrm{CH}_{3}\right) \mathrm{C}_{3} \mathrm{H}_{7}\right)$ and 1-butene $\left(\mathrm{H}_{2} \mathrm{C}=\mathrm{CHC}_{2} \mathrm{H}_{5}\right)$ are possible exceptions (Frère, 1989) (remember, however, that altitudes of condensation may be overestimated in Frère 's model). If methane condenses in the troposphere near the cold trap and if a metastable phase is excluded then condensing methane may only join a solid phase. Indeed, pure condensed methane would be solid at altitudes $z$ above $3 \mathrm{~km}$ in Titan's troposphere (corresponding to temperatures below $90.7 \mathrm{~K}$ ). Taking into account the miscibility of methane and nitrogen, the equilibrium condensed phase of methane-nitrogen freezes for $z \geq 14 \mathrm{~km}$ (corresponding to temperatures below about $81 \mathrm{~K}$ ) (Kouvaris and Flasar, 1991). For some condensable species, the triple point temperature is so high that nucleation of supercooled liquid is probably ruled out (see $\S 4$ below for the problem of supercooling). For instance, the triple point temperatures of $\mathrm{C}_{2} \mathrm{H}_{2}$ and $\mathrm{HCN}$ are $192 \mathrm{~K}$ and $260 \mathrm{~K}$ while, if they condense, it should be at temperatures below $100 \mathrm{~K}$ and $130 \mathrm{~K}$ respectively (Sagan and Thompson, 1984). 
Raulin (1987) qualitatively estimated the solubility of various polymers (polyethylene $\left(\mathrm{CH}_{2}\right)_{\mathrm{n}}$, polyacetylene $\left(\mathrm{C}_{2} \mathrm{H}_{2}\right)_{\mathrm{n}}$, polymethacrylonitrile $\left(\mathrm{C}_{4} \mathrm{H}_{5} \mathrm{~N}\right)_{\mathrm{n}}$, $\mathrm{HCN}$ polymers $(\mathrm{HCN})_{n}$, polyacrylonitrile $\left.\left(\mathrm{C}_{3} \mathrm{H}_{3} \mathrm{~N}\right)_{n}\right)$ in a liquid mixture of methane, nitrogen and ethane. The properties of those polymers provide a basis for inferring the properties of tholins. Polyethylene alone was found to be soluble or partially soluble, in solutions with low methane and high ethane mole fractions ${ }^{2}$. McKay (1996) reports that tholins produced in a laboratory simulation are insoluble in liquid ethane. Titan tholins should be more closely represented by McKay 's simulation tholins (with elemental composition corresponding to $\mathrm{C}_{11} \mathrm{H}_{11} \mathrm{~N}_{2}$ ) or by polyacetylene or polymers containing nitrogen than by polyethylene (Chassefière and Cabane, 1995). Thus, Titan tholins are expected to be insoluble in liquid ethane or methane.

Therefore, as far as atmospheric nucleation processes are concerned, the case of Titan may be closer to that of the terrestrial polar mesopause. There, the temperature is sufficiently below zero that water vapor is expected to nucleate directly into ice, and supersaturations probably reach much higher values than in the troposphere to allow efficient nucleation (Keesee, 1989).

\section{The classical theory of nucleation and its limits}

In the following, we will try to make a point that predictions about nucleation on Titan are possible since physical quantities important for the efficiency of nucleation, surface free enthalpies of solids and contact angles, may be estimated. Those quantities appear in the "classical" theory of nucleation. Before we proceed to show how surface free enthalpies and contact angles may be evaluated $(\S 5)$, we recall in this section the bases and limitations of the classical theory. Descriptions are given in McDonald (1962), McDonald (1963), Zettlemoyer (1969) and Pruppacher and Klett (1978).

We consider the heterogeneous nucleation of a pure condensed phase (liquid or solid) on a solid nucleus, implemented in the spherical-cap model. In the event of nucleation of a liquid phase, we only consider the case of an insoluble nucleus. The model is based on the following idealisations. The nucleus is a sphere, with a homogeneous surface. The "embryo" of condensed material grows as a spherical cap resting on the nucleus. That embryo is described as a macroscopic object, with radius $r$, using thermodynamic quantities which are properly defined only for macroscopic systems: contact angle, surface free enthalpy and density.

An embryo which has the critical radius, $\mathrm{r}^{*}$, is in unstable equilibrium with respect to growth or evaporation. Due to fluctuations, there is a population of

${ }^{2}$ Correcting the misprint in Raulin (1987, bottom of page 77 ). The right result of the thermodynamic analysis may be seen in figure 4 of that article. 
embryos of various sizes, from isolated molecules adsorbed on nuclei up to sizes greater than the critical size $\left(\mathrm{r}^{*}\right)$. If the vapor is supersaturated, the classical nucleation theory supposes that the size distribution of embryos is in quasistationary state and, consequently, that there is a uniform flow of embryos along the size distribution, starting from isolated molecules up to some size r' beyond the equilibrium size $r^{*}$. This flow of embryos reaching the supercritical region (beyond $\mathrm{r}^{\prime}$, see figure 2) is the nucleation rate $J . J$ is a function of temperature $T$, saturation ratio $S$ ( $S$ is equal to the ambient partial pressure of the vapor divided by the equilibrium vapor pressure $\mathrm{p}_{\mathrm{s}}$ of the pure compound over a flat surface of the condensed state) and radius $r_{N}$ of the nuclei. Material properties that enter into $J$ are $v_{\mathrm{v}}$, the frequency of vibration of an adsorbed molecule normal to the surface, $\Delta \mathrm{G}_{\mathrm{d}}$, the free enthalpy of desorption of an adsorbed molecule, $\sigma_{c a}$, the surface free enthalpy of the condensed material against air, $\rho_{\mathrm{c}}$, the density of the condensed phase and $\theta$, the contact angle of condensed material on the nucleus. $\theta$ depends on the chemical natures of the condensed phase and nucleus, and in principle on the composition of the air, on the saturation ratio $S$ and the temperature $T$, but is independent of the radii $r$ and $\mathrm{r}_{\mathrm{N}} . \theta$ is between 0 (perfectly wettable nucleus) and $\pi$ (unwettable nucleus). $\theta$ is related to the surface free enthalpies of the nucleus - air $\left(\sigma_{\mathrm{Na}}\right)$, nucleus condensed material $\left(\sigma_{\mathrm{Nc}}\right)$, and condensed material - air $\left(\sigma_{\mathrm{ca}}\right)$ interfaces by Young's relation (see e. g. Israelachvili, 1991, § 15.3):

$m=\cos \theta=\frac{\sigma_{N a}-\sigma_{N c}}{\sigma_{c a}}$

The classical theory of nucleation supposes various properties which may be quite far from reality. In particular, the nucleability of aerosols is characterized by the contact angle. The contact angle, originally defined for a liquid drop on a liquid or solid surface, loses meaning when we study the nucleation of a solid phase on a solid surface. Moreover, it pertains to an average macroscopic behavior, while some experiments show that nucleation on a solid substrate is favored by surface heterogeneity of the substrate: topographic features or, more generally, the presence of isolated "active" sites (Pruppacher and Klett, 1978, pages 259 and 262, 263). However, the classical theory seems to be the only operational description for heterogeneous nucleation in the atmosphere of Titan, where many different species must be taken into account and where nuclei are poorly characterized (see also a recent application to Neptune's atmosphere by Moses et al. (1992); the theory is still used even for water in Earth's atmosphere, see $e$. g. Keesee (1989)). We may speculate that, in Titan's atmosphere, the first volatile species condensing on tholin nuclei occupy the "best" active sites on the surface of tholins. Those first species, forming a small volume of condensed material (in comparison with the volume of tholin nuclei), should also make the shape of aerosols more symmetrical by appearing preferentially in the cavities of aerosols. So nucleation after the condensation of the first species might be controlled less by active sites than by an average behavior of the aerosols surface. We may consider the quantity $m=\cos \theta$ which 
intervenes in the expression for the nucleation rate $J$ as a parameter of compatibility between nucleus and condensed phase, and we may use Young's relation to obtain a qualitative indication of the value of $m$.

We have mentioned above that the nucleation rate of the classical theory corresponds to a quasi-stationary distribution of embryos. When, for instance, the saturation ratio changes, the distribution adapts itself in a certain time $\tau_{\text {adapt }}$ (see e. g. Dunning, 1969; Sigsbee, 1969; Pruppacher and Klett, 1978, page 173), before reaching a quasi-stationary state. This characteristic time decreases from infinity as $S$ increases from $S=1$. On the other hand, in Titan's atmosphere, the characteristic time of evolution $\tau_{\text {evol }}$ of the ambient saturation ratio (which depends on the settling velocity of aerosols and the altitude profile of $S$ ) should remain finite in the region where $S$ is close to 1 . Therefore, in that region, the nucleation rate may not be equal to its quasi-stationary value. This discrepancy does not matter if $\tau_{\text {adapt }}$ has become much smaller than $\tau_{\text {evol }}$ by the time the nucleation rate is "observable". The effect of non-negligible transient effects would be a time lag in nucleation, so we may only underestimate the importance of the nucleation phenomena.

We note that the classical theory also provides us with a mean to estimate whether the solid phase or the supercooled liquid phase of a species nucleates on an insoluble nucleus. This is done by comparing the magnitude of the corresponding nucleation rates (Dunning, 1969; Keesee, 1989). The presence of a liquid phase on aerosols in the lower stratosphere would have important implications for condensation of following species, which could be directly mixed into the liquid, without any nucleation barrier. To calculate the nucleation rate of a supercooled liquid, we need to extend the vapor-liquid equilibrium pressure curve $\mathrm{p}_{\text {liq }}(\mathrm{T})$ to temperatures below the triple point temperature $\mathrm{T}_{\mathrm{t}}$. If $T<\mathrm{T}_{\mathrm{t}}$ then $\mathrm{p}_{\text {liq }}(\mathrm{T})$ is greater than the vapor-solid equilibrium pressure $\mathrm{p}_{\text {sol }}(\mathrm{T})$. The nucleation of supercooled liquid is possible if the gas phase is supersaturated not only with respect to $\mathrm{p}_{\text {sol }}$ but also to pliq. Moreover, we need the surface tension, the density, and the contact angle of the supercooled liquid. The higher surface free enthalpy of the solid tends to make its nucleation rate smaller. This effect is balanced by the higher saturation ratio with respect to the solid phase.

\section{Required physical parameters: surface free enthalpies and contact angles}

As we can find experimental data on neither the surface free enthalpies of interfaces involving a solid phase, nor the contact angles, for the condensable species of Titan's atmosphere, we need theoretical or semi-empirical evaluations of those quantities. Although we will not be able here to reach numerical values, we intend to show that surface free enthalpies of solids and contact angles may be linked to other material properties which can clearly be measured in the laboratory. More precisely, bringing together results from surface physics and analyses on the water substance (motivated by the study of nucleation in Earth's 
atmosphere), we first pick out semi-empirical estimations (from latent heats and the liquid surface tension) which are quite easy to implement but have unknown respective validity for species of Titan's atmosphere. We need to test those semiempirical relations, for instance on $\mathrm{CH}_{4}$ for application to other alkanes, on $\mathrm{HCN}$ for application to other nitriles, etc. Hence, we suggest another way (the calculation of Hamaker constants) to find surface free enthalpies of solids and contact angles, which we think may give trustworthy values. As the experimental data necessary to compute Hamaker constants are more difficult to obtain than latent heats and liquid surface tensions (but definitely within reach), the method must probably be used only for reference species, in conjunction with the easier semi-empirical estimations.

The surface free enthapy of a solid in equilibrium with its pure vapor or with inert air (i. e. not adsorbed) is (e. g. Israelachvili, 1991, $\S 15.1$ ):

$\sigma=\frac{1}{2} W_{c}$

where $W_{c}$ is the energy of cleavage per unit area, or work needed to separate unit areas of the solid from contact to infinity. $\mathrm{W}_{\mathrm{c}}$ may be estimated in two ways: from the latent heats and the liquid surface tension, or from the Hamaker constant.

\subsection{Surface free enthalpy of a solid from the latent heats and the surface tension of the liquid}

$\mathrm{W}_{\mathrm{c}}$ may be written as the product of the surface density of molecules of the solid $\left(\mathrm{n}_{\text {surf,sol }}\right)$ and the bonding energy per molecule $(E) . E$ is the energy of interaction of a molecule with all the molecules on the other side of the cleavage plane. It may also be considered as the difference in bonding energy between a molecule in the bulk of the solid and a molecule at the surface. One may reasonably estimate $E$ as half the bonding energy of a molecule in the bulk, viz. (McDonald, 1953; Pruppacher and Klett, 1978, page 121; Adamson, 1990, § VII$3 \mathrm{E})$ :

$E=\frac{1}{2} \frac{L_{s u b}}{N_{A}}$

where $\mathrm{L}_{\mathrm{sub}}$ is the molar latent heat of sublimation and $\mathrm{N}_{\mathrm{A}}$ is the Avogadro number. We obtain:

$\sigma_{\text {sol }}=\frac{1}{4} \frac{L_{\text {sub }}}{N_{A}} n_{\text {surf, sol }}$

However, this derivation has not taken into account surface relaxation after cleavage. We actually expect $\sigma_{\text {sol }}$ to be lower than the value for a "fresh" surface, $\sigma_{\text {sol }}$, calculated by equation (10). McDonald (1953) suggests relating the liquid and solid surface relaxation by:

$\sigma_{\text {sol }} f-\sigma_{\text {sol }}=\left(\sigma_{\text {liq }} f \frac{n_{\text {surf,sol }}}{n_{\text {surf,liq }}}-\sigma_{\text {li } q}\right) \frac{L_{\text {vap }}}{L_{\text {sub }}}$

where $\sigma_{\text {liq }} f$ is calculated with the molar latent heat of vaporization, $L_{v a p}$, in the 
same way as $\sigma_{\text {sol }}{ }^{\mathrm{f}}$.

Thus:

$\sigma_{\text {sol }}=\frac{1}{4} \frac{L_{\text {sub }}}{N_{A}} n_{\text {surf, sol }}-\left(\frac{1}{4} \frac{L_{v a p}}{N_{A}} n_{\text {surf, sol }}-\sigma_{\text {liq }}\right) \frac{L_{v a p}}{L_{\text {sub }}}$

McDonald introduces in equation (11) the ratio of the latent heats of

vaporization and sublimation following the idea that the surface of the liquid - a phase with short-range order - is less constrained than the surface of the solid -

a phase with long-range order -, leading to a more important liquid relaxation. Pruppacher and Klett (1978) implement this idea in a different manner and write:

$\frac{\sigma_{\text {sol }}^{f}}{\sigma_{\text {sol }}}=\frac{\sigma_{\text {liq }}^{f}}{\sigma_{\text {liq }}} \times \frac{n_{\text {surf,sol }}}{n_{\text {surf,liq }}} \times \frac{L_{\text {vap }}}{L_{\text {sub }}}$

which comes down to:

$\sigma_{\text {sol }}=\left(\frac{L_{\text {sub }}}{L_{\text {vap }}}\right)^{2} \sigma_{l i q}$

(Actually, we guess this was the reasoning of Pruppacher and Klett since the corresponding passage in their section 5.7.1 seems very unclear to us. In particular, Pruppacher and Klett do not seem to recognize that McDonald revises the fresh surface value by subtracting a correction rather than introducing a multiplicative factor.) The value for water ice computed from equation (14) is closer to the experimental value than the value computed from equation (12). Adamson (1990, page 313) notes that the surface free enthalpies of the solid and the liquid near the triple point generally are in the proportion:

$\sigma_{\text {sol }}\left(T_{t}\right)=\frac{L_{\text {sub }}\left(T_{t}\right)}{L_{\text {vap }}\left(T_{t}\right)} \sigma_{l i q}\left(T_{t}\right)$

A choice between those semi-empirical correlations (equations (12), (14) and (15)), for the species we are interested in, can be made from the prediction of $\sigma_{\text {sol }}$ through the Hamaker constant.

\subsection{Surface free enthalpies from the Hamaker constant}

The Hamaker constant is a quantity which characterizes van der Waals interactions between macroscopic bodies (e. g. Israelachvili, 1991, chapter 11; Bowen and Jenner, 1995). The Hamaker constant $\mathrm{A}_{132}$, for interaction of media 1 and 2 across medium 3 , depends on the nature of the three media involved and on their thermodynamic state, but not on their shape or geometric arrangement. For instance, the van der Waals energy of interaction between two identical infinite walls (medium 1) separated by a medium 2 of thickness $D$ is, per unit area of one of the walls (see Israelachvili, 1991, chapter 11):

$W(D)=\frac{A_{121}}{12 \pi D^{2}}$

The energy $\mathrm{W}_{\mathrm{c}}$ required to cleave a unit area of solid (or liquid) 1 , $i$. $e$. to pull 
two surfaces of the solid apart from intermolecular contact $\left(\mathrm{D}=\mathrm{D}_{0}\right)$ to infinite distance, in vacuum or air, is (Israelachvili, 1991):

$W_{c}=\frac{A_{11}}{12 \pi D_{0}^{2}}=2 \sigma_{1}$

where $\sigma_{1}$ is the surface free enthalpy of the solid-air (or solid-vacuum) interface, and $\mathrm{A}_{11}$ is the Hamaker constant for interaction of 1 with itself, across air (or vacuum). Israelachvili (1991, § 11.10) finds that, with the "universal" value: $\mathrm{D}_{0}$ $=0.165 \mathrm{~nm}$, equation (17) yields reliable surface free enthalpies, within $20 \%$ of measured values, for ordinary solids and liquids, excluding only highly polar $\mathrm{H}-$ bonding species (like methanol $\mathrm{CH}_{3} \mathrm{OH}$, glycol $\mathrm{HO}\left(\mathrm{CH}_{2}\right)_{2} \mathrm{OH}$, water, glycerol $\mathrm{HOCH}_{2} \mathrm{CH}(\mathrm{OH}) \mathrm{CH}_{2} \mathrm{OH}, \mathrm{H}_{2} \mathrm{O}_{2}$, formamide $\mathrm{HC}\left(\mathrm{NH}_{2}\right) \mathrm{O}$ ) (and excluding metals).

The Hamaker constant is calculated on the basis of the Lifshitz theory (see Israelachvili, 1991, $\S 11.3)$. A sufficient approximation of the non-retarded value is (Israelachvili, 1991):

$A_{121}=\frac{3}{2} k_{B} T \sum_{n=0}^{+\infty}\left[\frac{\varepsilon_{1}\left(i v_{n}\right)-\varepsilon_{2}\left(i v_{n}\right)}{\varepsilon_{1}\left(i v_{n}\right)+\varepsilon_{2}\left(i v_{n}\right)}\right]^{2}$

where $\varepsilon_{1}$ and $\varepsilon_{2}$ are the complex relative dielectric permittivities of the two media (note that $\varepsilon(i v)$ is a real number), the frequency $v_{n}$ is given by:

$v_{n}=n \frac{2 \pi k_{B} T}{h}$

( $h$ is the Planck constant) and the primed symbol of summation indicates that the zero frequency term $(\mathrm{n}=0)$ is multiplied by one-half. The static term (zero frequency) in $A_{121}$ includes the Keesom and Debye interactions, while the nonstatic $(v>0)$ part of $A_{121}$ is due to the London interaction. $\varepsilon_{2} \equiv 1$ if medium 2 is vacuum or air, but the case of two condensed phases is also of interest, to compute their interfacial free enthalpy (see equation (26) below). Israelachvili (1991) gives reduced expressions of $\mathrm{A}_{121}$ for simple functional forms of $\varepsilon$ (iv) (as the form in equation (20) below).

The function $\varepsilon$ (iv) is related to the absorption spectrum, through the imaginary part $\varepsilon^{\prime \prime}(v)$ of $\varepsilon(v)$, by the Kramers-Kronig relation (see Hough and White, 1980):

$\varepsilon(i v)=1+\frac{2}{\pi} \int_{0}^{+\infty} \frac{v^{\prime} \varepsilon^{\prime \prime}\left(v^{\prime}\right)}{v^{\prime 2}+v^{2}} d v^{\prime}$

Thus, to compute the Hamaker constant, one needs to know, for each species considered, in the desired phase (generally solid for our study of Titan), at the desired temperature, the static dielectric permittivity and, in principle, the complete absorption spectrum.

In practice, instead of the whole absorption spectrum, the information required for sufficient accuracy on the Hamaker constant may be narrowed down as follows. For the study of Titan's lower atmosphere, we may consider temperatures between 70 and $150 \mathrm{~K}$, so the first non-zero frequency $v_{1}$ in 
equation (18) is in the middle infrared (corresponding wavelength between 15 $\mu \mathrm{m}$ and $33 \mu \mathrm{m})$. As the dielectric permittivities are sampled every $\Delta v=v_{1}$ (equation (18)), the ultraviolet contribution to the Hamaker constant is predominant. Therefore, the inaccuracy on the infrared contribution to the sum in equation (18) usually is of no importance. (There may be "pathological" cases when media 1 and 2 are both condensed phases, and their ultraviolet spectra are very similar, but not their radio or infrared spectra.) Let $\mathrm{n}_{\mathrm{vis}}$ be the real part of the refractive index, in the visible. For a "medium 1 - air - medium 1" system, the radio and infrared absorption may be neglected if $\left(\varepsilon_{1}(0)-\mathrm{n}_{\left.\mathrm{vis}, 1^{2}\right)}\right.$ is lower than, or of the order of 0.1 (see Hamaker constants calculations in Hough and White, 1980). If the difference $\left(\varepsilon_{1}(0)-\mathrm{n}_{\left.\mathrm{vis}, 1^{2}\right)}\right.$ is greater than that, and if a significant part of that difference is due to infrared absorption bands, then the frequencies of those bands and their relative integrated intensities are needed (Hough and White, 1980).

On the other hand, as concerns the ultraviolet absorption spectrum, the Hamaker constant is quite sensitive to the frequencies and relative integrated intensities of the electronic bands. If only one electronic absorption band, at a frequency $v_{\mathrm{e}}$, is noteworthy (responsible for most of the difference $\left(\mathrm{n}_{\mathrm{vis}}{ }^{2}-1\right)$ ), and the infrared absorption has been neglected, then an adequate representation of the function $\varepsilon(i v)$, in the non-static terms of equation (18), is (Hough and White, 1980):

$$
\varepsilon(i v)=1+\frac{n_{v i s}{ }^{2}-1}{1+\frac{v^{2}}{v_{e}{ }^{2}}}
$$

If the ultraviolet absorption spectrum is not available but is known to be simple (only one noteworthy band) then the values $\mathrm{n}_{\mathrm{vis}}$ and $v_{\mathrm{e}}$ to be set in equation (20) may be obtained, with very good accuracy, from a Cauchy plot (Hough and White, 1980; Bowen and Jenner, 1995). The Cauchy plot is drawn from the variation of the real refractive index with frequency in the visible.

In short, to compute Hamaker constants, the most important data, for each species, is the ultraviolet absorption spectrum of the solid phase. For lack of the ultraviolet spectrum, data on the real visible refractive index of the solid phase provide a workable alternative. However, note that sufficient accuracy to retrieve the wavelength dependence of the refractive index in the visible is then needed. The static dielectric permittivity of the solid phase is also desirable.

Preliminary results (on methane: Khare et al., 1990) are already available, which do not allow us to calculate contact angle values (low accuracy on the visible refractive index) but show that the required data are within reach of laboratory experiments. 


\subsection{Influence of adsorption}

The methods mentioned above can not take into account the effect on surface free enthalpy of adsorption of gas. The surface tension of liquids is little affected by adsorption of gas at ordinary pressures. Adsorption of a foreign species on a solid surface reduces the surface free enthalpy by the amount (Adamson, 1990, $\S \mathrm{X}-3 \mathrm{~B})$ :

$\pi(p)=\sigma(p=0)-\sigma(p)=\frac{R T}{\Sigma} \int_{0}^{p} n\left(p^{\prime}\right) \frac{d p^{\prime}}{p^{\prime}}$

where $p$ is the gas phase partial pressure of the adsorbed species, $\Sigma$ is the area of the solid surface, $\mathrm{n}(\mathrm{p})$ is the number of moles adsorbed when the partial pressure is $p$, and $\sigma(\mathrm{p})$ is the corresponding surface free enthalpy. The quantity $\pi$ is known as the film pressure (or surface, or spreading pressure). When more than one gas is adsorbed, $\pi$ is the sum of the partial film pressures, defined by equation (21), with each species treated separately. Adamson (1990, table X-2) gives some values of film pressures, when $p$ equals the saturation vapor pressure. Typical values range up to $100 \mathrm{~mJ} \mathrm{~m}^{2}$.

\subsection{Interface between two condensed phases}

If interactions between two condensed phases 1 and 2 are dominated by London forces (regardless of the internal interactions in each phase) then the surface free enthalpy of the interface may be approximated by (see Adamson, 1990, page 407; Israelachvili, 1991, page 316):

$\sigma_{12} \approx \sigma_{1}+\sigma_{2}-2 \sqrt{\sigma_{1}^{L} \sigma_{2}{ }^{L}}$

where $\sigma_{\mathrm{i}} \mathrm{L}$ is the contribution of London forces to the surface free enthalpy of medium $i$ (against air).

$\sigma_{\mathrm{i}}^{\mathrm{L}}$ may be estimated from the London term in the Hamaker constant:

$\sigma_{i}^{L}=\frac{A_{i i}{ }^{L}}{24 \pi D_{0}^{2}}$

(cf. Fowkes (1971) and the example of water in Adamson (1990, § X-6B) and Israelachvili (1991, page 316)).

If, moreover, internal interactions in media 1 and 2 are mainly London interactions too, then:

$\sigma_{\mathrm{i}}^{\mathrm{L}} \approx \sigma_{\mathrm{i}}$

$\sigma_{12} \approx \sigma_{1}+\sigma_{2}-2 \sqrt{\sigma_{1} \sigma_{2}}$

and $\sigma_{\mathrm{i}}$ may be calculated from equation (17). Alternatively, since we have the combining relation:

$A_{121} \approx A_{11}+A_{22}-2 \sqrt{A_{11} A_{22}}$

for media in which London forces dominate (Israelachvili, 1991, § 11.9), $\sigma_{12}$ 
may be calculated directly from:

$\sigma_{12}=\frac{A_{121}}{24 \pi D_{0}^{2}}$

\subsection{Contact angle}

Considering again a spherical cap of condensed matter resting on a solid nucleus, let us call $\sigma_{\mathrm{c}}$ and $\sigma_{\mathrm{N}}$ the surface free enthalpies of condensed phase and nucleus against their pure respective vapors. From equation (21), we have:

$\left\{\begin{array}{l}\sigma_{c a}=\sigma_{c}-\pi / c \\ \sigma_{N a}=\sigma_{N}-\pi / N\end{array}\right.$

where $\pi / \mathrm{c}$ and $\pi / \mathrm{N}$ are the total film pressures on the condensed material and nucleus respectively $(\pi / \mathrm{c}$ should be small if the condensed phase is liquid). (Note that the presence of adsorbed molecules of the condensing species on the nucleus is inherent to the classical theory of heterogeneous nucleation.) If the interactions between the nucleus and condensed phase are mainly London forces then, using equations (22) and (27), the Young equation (7) becomes:

$\cos \theta=-\frac{\pi_{/ N}}{\sigma_{c}-\pi_{/ c}}-\frac{\sigma_{c}}{\sigma_{c}-\pi_{/ c}}+2 \frac{\sqrt{\sigma_{N}^{L} \sigma_{c}^{L}}}{\sigma_{c}-\pi_{/ c}}$

If we neglect the film pressures, we obtain what has been called the GirifalcoGood-Fowkes-Young equation (Adamson, 1990, equation (X-48)):

$\cos \theta=-1+2 \frac{\sqrt{\sigma_{N}^{L} \sigma_{c}^{L}}}{\sigma_{c}}$

If the internal interactions in the nucleus and the condensed phase are mainly London interactions too, then:

$\sigma_{\mathrm{N}} \geq \sigma_{\mathrm{c}} \Rightarrow \theta=0$

$\sigma_{N}<\sigma_{c} \Rightarrow \cos \theta=-1+2 \sqrt{\frac{\sigma_{N}}{\sigma_{c}}}$

and the nucleus is partially wettable $(\theta \in] 0, \pi[)$.

Using relation (30), we may relate the uncertainty on surface free enthalpies to the uncertainty on contact angle. For instance, if the relative uncertainty in $\sigma_{\mathrm{N}}$ and $\sigma_{\mathrm{c}}$ is the same:

$\delta=\max \left|\frac{\sigma_{e s t}-\sigma_{t r}}{\sigma_{t r}}\right|=\max \left|\frac{\Delta \sigma}{\sigma_{t r}}\right|$

(subscripts "est" and "tr" for estimated and true values respectively), let us define $\alpha$ as:

$\alpha=\sqrt{\frac{1-\delta}{1+\delta}}$

Then the true value of the contact angle, $\theta_{\mathrm{tr}}$, is bounded by $\theta_{\mathrm{tr}, \min }$ and $\theta_{\mathrm{tr}, \max }$, such that: 


$$
\begin{aligned}
& \cos \theta_{t r, \text { max }}=\alpha\left(1+\cos \theta_{\text {est }}\right)-1 \\
& \left\{\begin{array}{l}
\cos \theta_{\text {est }}<2 \alpha-1 \Rightarrow \cos \theta_{t r, \text { min }}=\frac{1+\cos \theta_{\text {est }}}{\alpha}-1 \\
\cos \theta_{\text {est }} \geq 2 \alpha-1 \Rightarrow \cos \theta_{t r, \text { min }}=1
\end{array}\right.
\end{aligned}
$$

Equations (31) and (32) are plotted in figure 3, with $\delta=20 \%$, which is the value found by Israelachvili (1991) for estimation of surface free enthalpies through the Hamaker constant (see $\S 5.2$ above).

\section{Effects of finite nucleation rates on Titan: a first approach}

\subsection{The idea of a 'critical nucleation rate'}

Once we are able to compute the nucleation rate as a function of aerosol and gas properties, we may look for a simple criterion to know whether a calculated value is important or negligible. An important nucleation rate produces an important number density of supercritical embryos of condensed phase in a small time scale. So the choice of a critical value of the nucleation rate depends on what are considered a critical number density of embryos and a critical time scale, which may vary with the physical system considered, whether a cloud chamber experiment, or water in the terrestrial atmosphere, or the lower atmosphere of Titan. Moses et al. (1992) adopt: $\mathrm{J}_{\mathrm{cr}}=10^{-2} \mathrm{~cm}^{-3} \mathrm{~s}^{-1}$, following Keesee (1989), who states that rates of order $10^{-3}-10^{-2} \mathrm{~cm}^{-3} \mathrm{~s}^{-1}$ are thought to be necessary to produce a visible cloud in Earth's atmosphere. Here we try to obtain a critical value adapted to the case of Titan.

For a given profile of the mole fraction of the nucleating species, and for a given single-size aerosol distribution (radius $\mathrm{r}_{\mathrm{N}}(\mathrm{z})$, number density $\tilde{\mathrm{N}}(\mathrm{z})$, settling velocity $\mathrm{v}_{\mathrm{S}}(\mathrm{z})(<0)$, neglecting diffusion of aerosols), we can compute the number of supercritical embryos accumulated on a layer of aerosols, since the time when that layer crossed the $S=1$ level. Let us call $n$ the number of aerosols per unit area in the layer (independent of altitude, assuming plane-parallel geometry), and $\mathrm{n}_{\text {free }}$ the number of those aerosols, per unit area, that do not carry a (supercritical) embryo. If an aerosol may bear only one embryo then the variation of $\mathrm{n}_{\text {free }}$ corresponding to a variation $d z$ in altitude is:

$d n_{\text {free }}=-J \frac{d z}{v_{s}} n_{\text {free }}$

( $z$ increases upward and $\mathrm{v}_{\mathrm{S}}$ is negative.) ( $C f$. a similar calculation, for the median freezing temperature of a population of supercooled liquid drops, in Pruppacher and Klett (1978, pages 179 and 180).) Thus:

$n_{\text {free }}(z)=n \exp \left[\int_{z}^{z_{1}} \frac{J\left(z^{\prime}\right)}{v_{S}\left(z^{\prime}\right)} d z^{\prime}\right]$

where $\mathrm{z}_{1}$ is the saturation altitude: $\mathrm{S}\left(\mathrm{z}_{1}\right)=1$, and $J$ is the nucleation rate per nucleus. Now we may say that the main part of the nucleation period for the layer of interest is over (and the condensation period proper begins) at the 
altitude $\mathrm{z}_{\mathrm{cr}}$ where half the aerosols have received an embryo:

$$
\int^{z_{1}} \frac{J(z)}{\left|v_{s}(z)\right|} d z=\ln 2
$$

$z_{c r}$

If we only need to know $\mathrm{z}_{\mathrm{cr}}$ with an uncertainty $\Delta \mathrm{z}$ not too small (see below) then, taking advantage of the rapid increase of $J$ with decreasing altitude, we may define:

$J_{c r}=\frac{\left|v_{s}\right|}{\Delta z}$

and the nucleation is "observable" at the approximate altitude (i. e. to within $\Delta \mathrm{z}$ ) where $J=\mathrm{J}_{\mathrm{cr}} . \Delta \mathrm{z}$ must be greater than or of the order of $\left|\mathrm{J} / \frac{\mathrm{dJ}}{\mathrm{dz}}\right|$ at $\mathrm{z}_{\mathrm{cr}}$ to ensure that the nucleating history of the layer (between $\mathrm{z}_{1}$ and $\mathrm{z}_{\mathrm{cr}}$ ) is at most of the same importance than what happens in the region $\left[z_{c r}-\Delta z, z_{c r}\right]$.

With our definition, nucleation is at a critical point where the number density of aerosols bearing condensed matter is of the same order of magnitude than the total number density of aerosols $\tilde{\mathrm{N}}$. This does not measure the importance of condensation nor the observable quality of the cloud: if $\tilde{\mathrm{N}}$ is very small and nucleation is critical (or more than critical) then the corresponding number of aerosols bearing condensed matter is very small too. Thus, the critical nucleation rate here should be understood simply as the rate at which the barrier for nucleation on aerosols is overcome.

If the aerosols do not receive condensed matter in the lower stratosphere nor in the troposphere, then their radius $r_{N}$ below $50 \mathrm{~km}$ (approximately the region where methane can be saturated or supersaturated) should be between 0.2 and $0.5 \mu \mathrm{m}$ (in a spherical drop aerosol model) and their number density between 10 and $10^{3} \mathrm{~cm}^{-3}$ (McKay et al., 1989, figure 3; Cabane et al., 1992, figure 4a; Cabane et al., 1993, figure 3). Using $\rho_{\mathrm{N}}=1 \mathrm{~g} \mathrm{c}$ h for the density of tholin material, $\left|\mathrm{v}_{\mathrm{s}}\right|$ is between 2 and $30 \mu \mathrm{m} \mathrm{s}^{-1}$ below $50 \mathrm{~km}$. With $\Delta \mathrm{z}=2 \mathrm{~km}$ (the sampling interval for the pressure and temperature profiles), $J_{\mathrm{cr}}$ is about $10^{-9} \mathrm{~s}^{-}$ $1-10^{-8} \mathrm{~s}^{-1}$ for the $[0,50 \mathrm{~km}]$ region (corresponding to $10^{-8} \mathrm{~cm}^{-3} \mathrm{~s}^{-1}-10^{-5} \mathrm{~cm}^{-3}$ $\mathrm{s}^{-1}$ ). If, on the other hand, species other than methane readily condense in the stratosphere then, at the tropopause, $\mathrm{r}_{\mathrm{N}}$ could be as large as $2 \mu \mathrm{m}$, and $\left|\mathrm{v}_{\mathrm{s}}\right| \approx 2 \times$ $10^{-4} \mathrm{~m} \mathrm{~s}^{-1}$ (Frère, 1989, table $\mathrm{C} 4$ ), so: $\mathrm{J}_{\mathrm{cr}}=10^{7} \mathrm{~s}^{-1}$. Figure 4 shows the critical saturation ratio $\mathrm{S}_{\mathrm{cr}}\left(\mathrm{J}\left(\mathrm{S}_{\mathrm{cr}}\right)=\mathrm{J}_{\mathrm{cr}}\right)$ for methane at the tropopause, with the former hypothesis. Calculations for figures 4 to 7 assume, following Moses et al. (1992), that embryos of condensed phase grow directly from vapor phase molecules rather than from adsorbed molecules, $h v_{\mathrm{v}}=\mathrm{k}_{\mathrm{B}} \mathrm{T}$ (see $\S 4$ ) and $\Delta \mathrm{G}_{\mathrm{d}}=$ $0.18 \mathrm{eV}$ (as reported by Seki and Hasegawa (1983) for water on silicates). For a study of sensitivity to the $\Delta \mathrm{G}_{\mathrm{d}}$ parameter, one can use the maximum observed enthalpies of physisorption given by Atkins (1990, table 29.1) for $\mathrm{C}_{2} \mathrm{H}_{2} \quad(0.39$ $\mathrm{eV}), \mathrm{C}_{2} \mathrm{H}_{4}(0.35 \mathrm{eV}), \mathrm{CH}_{4}(0.22 \mathrm{eV}), \mathrm{CO}, \mathrm{H}_{2} \mathrm{O}$ and $\mathrm{N}_{2}$. 
We see that if a saturation ratio equal to 2 is to be sustained without significant nucleation (see $\S 3$ above), then the contact angle must be greater than about $40^{\circ}$. We check that $\Delta \mathrm{z}$ is greater than $\left|\mathrm{J} / \frac{\mathrm{dJ}}{\mathrm{dz}}\right|$ at $\mathrm{z}_{\mathrm{cr}}$ in the following way: we take $\theta=40^{\circ}$ and a constant mixing ratio of methane $(4.7 \%)$, such that $S=2$ at the tropopause, then $\left|\mathrm{J} / \frac{\mathrm{dJ}}{\mathrm{dz}}\right|(40 \mathrm{~km})$ is of the order of $0.1 \mathrm{~km}$ (see figure 5). An almost constant mixing ratio in the $[0,50 \mathrm{~km}]$ region is obtained when one supposes that the eddy diffusion coefficient is of the order of $0.1 \mathrm{~m}^{2} \mathrm{~s}^{-1}$ and that the gas flux of methane is constant, of the order of $10^{14} \mathrm{~m}^{-2} \mathrm{~s}^{-1}$ (the chemical loss flux). This implies that there is no methane condensation.

This critical rate approach is limited in that we need to start from a profile of the saturation ratio to infer the altitude where condensation proper begins. In their study of Neptune's atmosphere, Moses et al. (1992) use saturation ratios from a photochemical model without condensation. If the critical rate is exceeded for a certain species (other than methane), then condensation will not only modify the saturation ratio below the critical level but also above it, due to gas diffusion processes. This in turn alters the critical level. Thus, for species other than methane, we expect the critical levels calculated in that way, for a particular mode of nucleation (and particular values of $\mathrm{r}_{\mathrm{N}}$ and $\theta$ ), to be upper limits of the actual condensation level. As for methane, prediction of the location of condensation is even more difficult. Although gaseous methane is replenished from the surface, we cannot say that condensation occurs only above the lower critical level (the level where $J$ crosses $J_{c r}$ below the cold trap), as Moses et al. suggest, because condensed methane which would appear above the critical level, would keep growing after settling below it. We may only speculate that at each altitude below the cold trap, the methane mole fraction $\mathrm{x}(\mathrm{z})$ is smaller than or equal to $\mathrm{x}_{\mathrm{cr}}(\mathrm{z})=\mathrm{S}_{\mathrm{r}}(\mathrm{z}) \mathrm{x}_{\mathrm{s}}(\mathrm{z})$, and $\mathrm{x}(\mathrm{z})$ above the cold trap is smaller than the minimum of $x_{c r}$ (see figure 6).

\subsection{Potential consequences}

Estimating the finite nucleation rates of the various species on Titan, we see that, as pointed out by Moses et al. (1992) for Neptune, the regions of effective condensation may be appreciably narrower than the saturation regions ( $c f$. the example of ethane in figure 7). The condensation of a species on aerosols can even be completely inhibited. For species other than methane, condensable in the lower stratosphere, the nucleation delay would lower the altitudes of condensation. So the order in which species successively condense on aerosols, which determines the nucleation rates through the contact angles, becomes itself unknown. Many scenarios can then be envisaged. For instance, aerosols with low surface energy, which do not nucleate efficiently any species without high supersaturation, could finally receive a layer of condensed material with 
higher surface energy, leading to the avalanche condensation of all supersaturated species.

Romani et al. (1993) put forward another potential consequence of nucleation difficulties. They describe the following scenario: the saturation ratio of a species grows until the nucleation rate reaches a high enough value; effective condensation starts and quickly depletes the highly supersaturated vapor phase; nucleation shuts down; the aerosols bearing condensed material settle out of the saturation region; the vapor phase is replenished, the saturation ratio builds up again. So the atmosphere could undergo periodic or, more generally, non stationary evolution. In order to get an idea of the time scale involved for such evolution in the case of methane, we may consider that the process would deplete the vapour phase between 10 and $30 \mathrm{~km}$ approximately. Indeed, take for instance the case where $\theta=35^{\circ}$ in figure 6: condensation is triggered where $x$ reaches $\mathrm{x}_{\mathrm{cr}}$, between 15 and $30 \mathrm{~km}$, but rain falls below $15 \mathrm{~km}$ so condensation on settling aerosols continues as long as the gas is supersaturated, down to about $10 \mathrm{~km}$. The time scale for the replenishing of the gas phase on a $20 \mathrm{~km}$ thick region is $\frac{(20 \mathrm{~km})^{2}}{\mathrm{~K}}$. That is of the order of 100 terrestrial years or greater if $\mathrm{K} \leqslant 0.1 \mathrm{~m}^{2} \mathrm{~s}^{-1}$ (Flasar et al., 1981). The gas replenishing time is a minimum time scale for the evolution of the troposphere in our scenario. Actually, the depleting of the gas phase (time scale of the order of $\frac{1}{4 \pi \mathrm{rDN}}$ [see equation (6)], smaller than about $10^{4} \mathrm{~s}$, corresponding to $r=0.2 \mu \mathrm{m}, \tilde{\mathrm{N}}=10 \mathrm{~cm}^{-3}$ and $D=2 \times$ $10^{-6} \mathrm{~m}^{2} \mathrm{~s}^{-1}$ ) and the settling out of aerosols bearing condensed material - time scale smaller than $10^{7} \mathrm{~s}$, corresponding to velocity $\left|\mathrm{v}_{\mathrm{s}}\right| \geqslant 1 \mu \mathrm{m} \mathrm{s}^{-1}$ (radius $\mathrm{r} \geqslant 10$ $\mu \mathrm{m}$; Toon et al., 1988) - are much quicker so gas replenishing should be the limiting process in such atmospheric evolution.

Last, there is the possibility of several modes in the aerosol distribution (both in size and in nature of the surface) coexisting at the same altitude. This can happen in non-stationary evolution, if aerosols with condensed matter catch up with aerosols which previously passed untouched through the saturation region. It can also be due simply to "just right" values of the nucleation rate: values which allow condensation on a part of the aerosol distribution which is not negligible nor overwhelming.

\section{Conclusion}

As has been previously suggested (Moses et al., 1992), nucleation may be difficult in the atmospheres of the outer solar system. The fundamental reason is that the stable condensed phases in saturation regions are solid, not liquid. One could then speculate on the existence of supercooled liquid, especially if soluble nuclei are present. However, the insolubility of Titan's tholins in non-polar hydrocarbons (McKay, 1996) is a negative element in this hypothesis, and allows 
that condensation of all species is inhibited everywhere but in the immediate vicinity of the surface. In particular, there may be significant methane supersaturation in the troposphere, either permanent or periodic, according to the contact angle of condensed methane on aerosols and to the surface relative humidity. This remains an open question.

Modeling the distribution of aerosols, nucleation and phase changes in Titan's lower atmosphere should indirectly offer new insight on atmospheric chemistry, the nature of Titan's surface and the temperature profile. Such modeling should help to deduce the profiles of cloud extinction and latent heat exchange, the amount of methane supersaturation, and whether some parts of the surface are washed by methane rain. Depending on methane supersaturation in the troposphere, the mixing ratio of methane in the stratosphere and above varies (up to $12 \%$ using background atmospheric profiles from Lellouch et al. (1990)), hence the depth of penetration of ultraviolet photons, hence the temperature and pressure where photochemistry takes place. Depending on the existence and properties of precipitation down to the surface, exposure of the icy bedrock may be explained by rain washing of elevated terrain, or volcanism or an impact event may be indicated (Smith et al., 1996). The approach by physical modeling of phase changes is complementary to studies of radiative transfer, which constrain cloud parameters and methane abundance from the Voyager IRIS spectra and the ground-based observations of geometric albedo. For instance, knowing that significant methane supersaturation and negligible cloud opacity provide a good fit (but not the only acceptable fit) to the emission spectrum in the $\left[200 \mathrm{~cm}^{-1}, 600 \mathrm{~cm}^{-1}\right.$ ] wave number range (Courtin et al., 1995), it remains to study the consistency of those two properties from the point of view of cloud physics on Titan.

We have shown that if methane nucleation is easy then the fluxes of methane in the troposphere may be much more important than the chemical destruction flux of methane and that latent heat exchange may have a noticeable influence on the thermal profile. This does not necessarily conflicts with observations which allow only a moderate global cloud optical depth. Indeed, the number density of methane crystals or drops may still be small. Including latent heat exchange in a thermal structure model can provide a new constraint in the investigation of the properties of the lower atmosphere.

For the description of heterogeneous nucleation, the classical theory has serious deficiencies, but is the only one simple and general enough to be used for Titan's atmosphere, which contains many different species, and poorly known nuclei. The quantities which control the magnitude of the nucleation rate, namely surface free enthalpy and contact angle, are not unattainable. To use the evaluation methods we have reported, the main data which remain to be assembled concerning the condensable species of Titan's atmosphere are adsorption behavior, the static dielectric permittivities of solid phases and their ultraviolet absorption spectra (or the spectral dispersion of their visible real 
refractive indices to extrapolate the ultraviolet absorption). The laboratory experiments which would yield those data are entirely feasible and the data would benefit other fields of research: the study of radiation transfer on Uranus, Neptune, Triton and Pluto (possible solid methane clouds), in comets and interstellar medium (possible solid $\mathrm{CH}_{4}$ and other solid hydrocarbons), reflection from the icy surfaces of solar system bodies, and the depth of penetration of energetic particles in comets or interstellar grains (Khare et al., 1990). The static dielectric permittivities would also be useful for the study of ion-induced nucleation on Neptune and Titan (see Moses et al., 1992).

The concept of a critical nucleation rate may show us that, beyond a specific value of contact angle, nucleation is completely inhibited in the whole atmosphere. However, we feel it is not able to predict (quantitatively) the shift in condensation altitudes, especially not in the case of methane. That would require, at least, a model coupling nucleation, gas diffusion, aerosol settling, and condensation proper.

Modeling nucleation on top of condensation may be very intricate because the order in which species nucleate and condense becomes unknown. One could consider as many contact angle parameters as there are pairs of species in the model. We see then the importance in estimating these contact angles.

\section{Acknowledgements}

This work was supported by a grant from the Centre National d'Etudes Spatiales, in the frame of a Huygens - IDS program. We thank the two referees, J. I. Moses and C. P. McKay, for many helpful comments, R. Courtin for discussion and N. Smith for improving the English. 


\section{$\underline{\text { References }}$}

Adamson, A. W. Physical Chemistry of Surfaces. 5th ed. John Wiley and Sons, New-York. 1990.

Atkins, P. W. Physical Chemistry. 4th ed. Oxford university press, Oxford. 1990 .

Awal, M. and Lunine, J. I. Moist Convective Clouds in Titan's Atmosphere. Geophys. Res. Lett. 21 (23), 2491-2494. 1994.

Bowen, W. R. and Jenner, F. The Calculation of Dispersion Forces for Engineering Applications. Adv. Colloid Interface Sci. 56, 201-243. 1995.

Cabane, M., Chassefière, E. and Israël, G. Formation and Growth of Photochemical Aerosols in Titan's Atmosphere. Icarus 96, 176-189. 1992.

Cabane, M., Rannou, P., Chassefière, E. and Israël, G. Fractal Aggregates in Titan's Atmosphere. Planet. Sp. Sci. 41 (4), 257-267. 1993.

Chassefière, E. and Cabane, M. Two Formation Regions for Titan's Hazes: Indirect Clues and Possible Synthesis Mechanisms. Planet. Sp. Sci. 43 (1/2), 91103. 1995.

Coll, P., Coscia, D., Gazeau, M.-C., Guez, L. and Raulin, F. Review and Latest Results of Laboratory Investigations of Titan's Aerosols. Orig. Life, in press. 1997.

Courtin, R., Gautier, D. and McKay, C. P. Titan's Thermal Emission Spectrum: Re-Analysis of the Voyager Infrared Measurements. Icarus 114, 144162. 1995.

Cousténis, A., Lellouch, E., Maillard, J. P. and McKay, C. P. Titan's Surface: Composition and Variability from the Near-Infrared Albedo. Icarus 118 (1), 87-104. 1995.

Dunning, W. J. General and Theoretical Introduction. In Nucleation. Zettlemoyer, A. C. (ed.). Marcel Dekker, New York. Pages 1-68. 1969.

Fink, U. and Larson, H. P. The Infrared Spectra of Uranus, Neptune and Titan from 0.8 to 2.5 Microns. Astrophys. J. 233, 1021-1040. 1979.

Flasar, F. M., Samuelson, R. E. and Conrath, B. J. Titan's Atmosphere:

Temperature and Dynamics. Nature 292, 693-698. 1981.

Fowkes, F. M. Predicting Attractive Forces at Interfaces. Analogy to Solubility

Parameter. In Chemistry and Physics of Interfaces-II. Ross, S. (ed.). American Chemical Society, Washington. Pages 153-167. 1971.

Frère, C. Etude physico-chimique de l'atmosphère, de l'aérosol et de l'océan de Titan. Thèse de 3ème cycle. University Paris 12. 1989.

Frère, C., Raulin, F., Israël, G. and Cabane, M. Microphysical Modeling of Titan's Aerosols: Application to the in Situ Analysis. Adv. Space Res. 10 (1), 159163. 1990.

Griffith, C. A. Evidence for Surface Heterogeneity on Titan. Nature 364, 511514. 1993.

Griffith, C. A., Owen, T. and Wagener, R. Titan's Surface and Troposphere, Investigated with Ground-Based, Near-Infrared Observations. Icarus 93, 362378. 1991. 
Hough, D. B. and White, L. R. The Calculation of Hamaker Constants from Lifshitz Theory with Applications to Wetting Phenomena. Adv. Colloid Interface Sci. 14, 3-41. 1980.

Israelachvili, J. N. Intermolecular and Surface Forces. 2nd ed. Academic Press, London. 1991.

Keesee, R. G. Nucleation and Particle Formation in the Upper Atmosphere. $J$. Geophys. Res. (Atm.) 94, 14683. 1989.

Khare, B. N., Thompson, W. R., Sagan, C., Arakawa, E. T., Bruel, C., Judish, J. P., Khanna, R. K. and Pollack, J. B. Optical Constants of Solid Methane. Proceedings of the First international conference on laboratory research for planetary atmospheres. Bowie state university, Bowie, Maryland. Fox, K., Allen Jr., J. E., Stief, L. J. and Quillen, D. T. (ed.). NASA Conference Publication. 3077. Pages 327-339. 1990.

Kirk, B. S. and Ziegler, W. T. A Phase-Equilibrium Apparatus for Gas-Liquid Systems and the Gas Phase of Gas-Solid Systems: Application to MethaneHydrogen from 66.88 to $116.53 \mathrm{~K}$ and up to 125 Atmospheres. In Advances in Cryogenic Engineering. Timmerhaus, K. D. (ed.). Volume 10, part 2. Plenum, New-York. Pages 160-170. 1965.

Kouvaris, L. C. and Flasar, F. M. Phase Equilibrium of Methane and Nitrogen at Low Temperature: Application to Titan. Icarus 91, 112. 1991.

Lara, L. M., Lorenz, R. D. and Rodrigo, R. Liquids and Solids on the Surface of Titan. Results of a New Photochemical Model. Planet. Sp. Sci. 42 (1), 5-14. 1994.

Lellouch, E. Atmospheric Models of Titan and Triton. Ann. Geophys. 8 (10), 653-660. 1990.

Lemmon, M. T., Karkoschka, E. and Tomasko, M. Titan's Rotational LightCurve. Icarus 113 (1), 27-38. 1995.

Liou, K.-N. Radiation and Cloud Processes in the Atmosphere: Theory, Observation, and Modeling. Oxford Monographs on Geology and Geophysics. Volume 20. Charnock, H., Dewey, J. F., Morris, S. C., Navrotsky, A., Oxburgh, E. R., Price, R. A. and Skinner, B. J. (series ed.). Oxford university press, Oxford. 1992.

Lorenz, R. D. The Life, Death and Afterlife of a Raindrop on Titan. Planet. Sp. Sci. 41 (9), 647-655. 1993a.

Lorenz, R. D. The Surface of Titan in the Context of ESA's Huygens Probe. ESA J. 17 (4), 275-292. 1993b.

Lorenz, R. D. Crater Lakes on Titan. Rings, Horseshoes and Bullseyes. Planet. Sp. Sci. 42 (1), 1-4. 1994.

Lunine, J. I. Does Titan Have an Ocean? A Review of Current Understanding of Titan's Surface. Rev. Geophys. 31 (2), 133-149. 1993.

McDonald, J. E. Homogeneous Nucleation of Supercooled Water Drops. J. Meteorol. 10, 416-433. 1953.

McDonald, J. E. Homogeneous Nucleation of Vapor Condensation. I. Thermodynamic Aspects. Am. J. Phys. 30, 870-877. 1962.

McDonald, J. E. Homogeneous Nucleation of Vapor Condensation. II. Kinetic Aspects. Am. J. Phys. 31, 31-41. 1963. 
McKay, C. P. Elemental Composition, Solubility and Optical Properties of Titan's Organic Haze. Planet. Sp. Sci. 44 (8), 741-747. 1996.

McKay, C. P., Pollack, J. B. and Courtin, R. The Thermal Structure of Titan's Atmosphere. Icarus 80 (1), 23-53. 1989.

McKay, C. P., Pollack, J. B. and Courtin, R. The Greenhouse and Antigreenhouse Effects on Titan. Science 253, 1118-1121. 1991.

Moses, J. I., Allen, M. and Yung, Y. L. Hydrocarbon Nucleation and Aerosol Formation in Neptune's Atmosphere. Icarus 99, 318-346. 1992.

Muhleman, D. O., Grossman, A. W. and Butler, B. J. Radar Investigation of Mars, Mercury, and Titan. Ann. Rev. Earth and Plan. Sci. 23, 337-374. 1995. Muhleman, D. O., Grossman, A. W., Slade, M. A. and Butler, B. J. The Surface of Titan and Titan's Rotation: What Is Radar Telling Us? Bull. Am. Astr. Soc. 24, 954-955. 1992.

Neff, J. S., Humm, D. C., Bergstralh, J. T., Cochran, A. L., Cochran, W. D., Barker, E. D. and Tull, R. G. Absolute Spectrophotometry of Titan, Uranus and Neptune: 3500 - 10,500 ̊. Icarus 60 (2), 221-235. 1984.

Pruppacher, H. R. and Klett, J. D. Microphysics of Clouds and Precipitation. Reidel, Dordrecht. 1978.

Raulin, F. Organic Chemistry in the Oceans of Titan. Adv. Space Res. 7 (5), 71 81. 1987.

Raulin, F., Bruston, P., Coll, P., Coscia, D., Gazeau, M.-C., Guez, L. and de Vanssay, E. Exobiology on Titan. J. Biol. Phys. 20, 39-53. 1994.

Romani, P. N., Bishop, J., Bézard, B. and Atreya, S. Methane

Photochemistry on Neptune: Ethane and Acetylene Mixing Ratios and Haze Production. Icarus 106, 442-463. 1993.

Sagan, C. and Thompson, W. R. Production and Condensation of Organic Gases in the Atmosphere of Titan. Icarus 59, 133-161. 1984.

Seinfeld, J. H. Atmospheric Chemistry and Physics of Air Pollution. Wiley Interscience, New-York. 1986.

Seki, J. and Hasegawa, H. The Heterogeneous Condensation of Interstellar Ice Grains. Astrophys. Space Sci.94, 177-189. 1983.

Sigsbee, R. A. Vapor to Condensed-Phase Heterogeneous Nucleation. In

Nucleation. Zettlemoyer, A. C. (ed.). Marcel Dekker, New York. Pages 151-224. 1969.

Smith, P. H., Lemmon, M. T., Lorenz, R. D., Sromovsky, L. A., Caldwell, J. J. and Allison, M. D. Titan's Surface, Revealed by HST Imaging. Icarus 119 (2), 336-349. 1996.

Summers, M. E. and Strobel, D. F. Photochemistry of the Atmosphere of Uranus. Astrophys. J. 346, 495-508. 1989.

Toon, O. B., McKay, C. P., Courtin, R. and Ackerman, T. P. Methane Rain on Titan. Icarus 75, 255-284. 1988.

Toon, O. B., McKay, C. P., Griffith, C. A. and Turco, R. P. A Physical Model of Titan's Aerosols. Icarus 95, 24-53. 1992.

Toublanc, D., Parisot, J. P., Brillet, J., Gautier, D., Raulin, F. and McKay, C. P. Photochemical Modeling of Titan's Atmosphere. Icarus 113 (1), 226. 1995. 
Yung, Y. L., Allen, M. and Pinto, J. P. Photochemistry of the Atmosphere of Titan: Comparison between Model and Observations. Astrophys. J. Supp. Ser. 55, 465-506. 1984.

Zettlemoyer, A. C. (ed.). Nucleation. Marcel Dekker, New-York. 1969. 


\section{Figure Captions}

Figure 1:

(a): Methane mole fraction.

The continuous flux profile corresponds to the methane gas fluxes $\Phi(\mathrm{z}=0) \approx 1.5$ $\times 10^{19} \mathrm{~m}^{-2} \mathrm{~s}^{-1}$ (see $\left.\S 2.1\right)$ and $\Phi=1.3 \times 10^{14} \mathrm{~m}^{-2} \mathrm{~s}^{-1}$ above the cold trap $(\approx 30$ $\mathrm{km}) . \mathrm{x}_{\mathrm{S}}$ is the saturation profile.

(b): Graphical resolution of the continuous flux problem.

$\Phi_{\mathrm{s}}$ is the methane gas flux corresponding to $\mathrm{x}_{\mathrm{s}} . \Phi_{\mathrm{surf}}\left(\mathrm{z}_{\mathrm{i}}\right)$ is the surface flux such that $x$ reaches $\mathrm{x}_{\mathrm{S}}$ at $\mathrm{z}_{\mathrm{i}}$ (see $\left.\S 2.1\right)$. The curves cross at $\mathrm{z}_{\mathrm{i}}{ }^{*} \approx 19 \mathrm{~km}$, for $\Phi \approx 1.5 \times 10^{19} \mathrm{~m}^{2} \mathrm{~s}^{-1}$.

Figure 2: Sketch of the distribution of embryos in quasi-stationary state.

The abscissa $k$ is the number of molecules in the embryo; $\mathrm{k}^{*}$ corresponds to the equilibrium radius $\mathrm{r}^{*}$; $\mathrm{k}^{\prime}$ is a number of molecules beyond $\mathrm{k}^{*}\left(\left(\mathrm{k}^{\prime}-\mathrm{k}^{*}\right)\right.$ may be of the order of $\mathrm{k}^{*}$ ) and corresponds to radius $\mathrm{r}^{\prime}$ (see $\S 4$ ). Below $\mathrm{k}^{\prime}$ is the quasistationary range. The ordinate $f_{k}$ is the number of embryos (per nucleus) containing $k$ molecules. $J$ is the flow of embryos along the size axis.

Figure 3: Uncertainty on the contact angle.

$\theta_{\text {est }}$ is the contact angle estimated from equation (30), $\theta_{\operatorname{tr}}$ is the true value. $\delta$ is the relative uncertainty on the surface free enthalpies $\sigma_{N}$ and $\sigma_{c}$ and is taken equal to $20 \%$. The point $\left(\theta_{\text {est }}, \theta_{\text {tr }}\right)$ must be in the region between the curves. This figure may be used together with figures 5 to 7 . For instance, considering the value of the critical nucleation rate, figure 5 shows that methane condensation should be completely inhibited everywhere for $\theta \geq 60^{\circ}$, and will definitely occur somewhere for $\theta \leq 50^{\circ}$, but is uncertain for intermediate $\theta$. Figure 3 then shows that the contact angle value $\theta_{\text {est }}$ will allow a conclusion concerning condensation of methane only if $\theta_{\text {est }}$ is greater than $80^{\circ}$ or very close to $0^{\circ}$.

Figure 4:

Critical saturation ratio $S_{c r}$ as a function of contact angle $\theta$, for methane at 71.1 $\mathrm{K}$ (tropopause temperature), on nuclei of radius $0.5 \mu \mathrm{m}$. The surface tension of liquid methane is used, as in Moses et al. (1992). The critical nucleation rate is about $10^{-9}-10^{-8} \mathrm{~s}^{-1}$ if the nucleation is to proceed within $\Delta \mathrm{z}=2 \mathrm{~km}$.

Figure 5:

Nucleation rate $J$ of methane as a function of altitude $z$ and contact angle, on nuclei of radius $0.5 \mu \mathrm{m}$. The mole fraction of methane is assumed to be constant, equal to $4.7 \%$, so that $S=2$ at the tropopause $(40 \mathrm{~km}) . S=1$ at $49 \mathrm{~km}$ and $9.6 \mathrm{~km}$. The figure illustrates the steepness of the nucleation rate increase above the level where $J$ crosses $\mathrm{J}_{\mathrm{cr}}$ (which is the tropopause if $\theta \approx 40^{\circ}$ ).

Figure 6:

Saturation-mole fraction $\left(\mathrm{x}_{\mathrm{S}}\right)$ and critical mole fractions $\left(\mathrm{x}_{\mathrm{cr}}\right)$ of methane for 
different contact angles $(\theta)$, assuming: $\mathrm{J}_{\mathrm{cr}}=1 \circledast \mathrm{s}^{-1}$ and $\mathrm{r}_{\mathrm{N}}=0.5 \mu \mathrm{m}$. The probable maximum mole fraction of methane $\left(x_{\max }\right)$ is also shown in the case: $\theta$ $=35^{\circ}$ and $\mathrm{x}(\mathrm{z}=0)=4.5 \%$.

Figure 7:

Nucleation rate $J$ of ethane as a function of altitude $z$ and contact angle $\theta$. The mole fraction of ethane in the gas phase is taken equal to $1.3 \times 10^{-5}$, the radius of nuclei is $0.5 \mu \mathrm{m}$. The surface tension of liquid ethane is used, as in Moses et al. (1992). A vertical line is drawn at $J=10^{-9} \mathrm{~s}^{-1}$, which may be regarded as a critical value: the upper intersection of one of the curves with that line gives the maximum altitude where effective condensation begins (see $\S$ 6.1). The saturation ratio is equal to 1 at $z=1.5 \mathrm{~km}$ and $z=62 \mathrm{~km}$. 

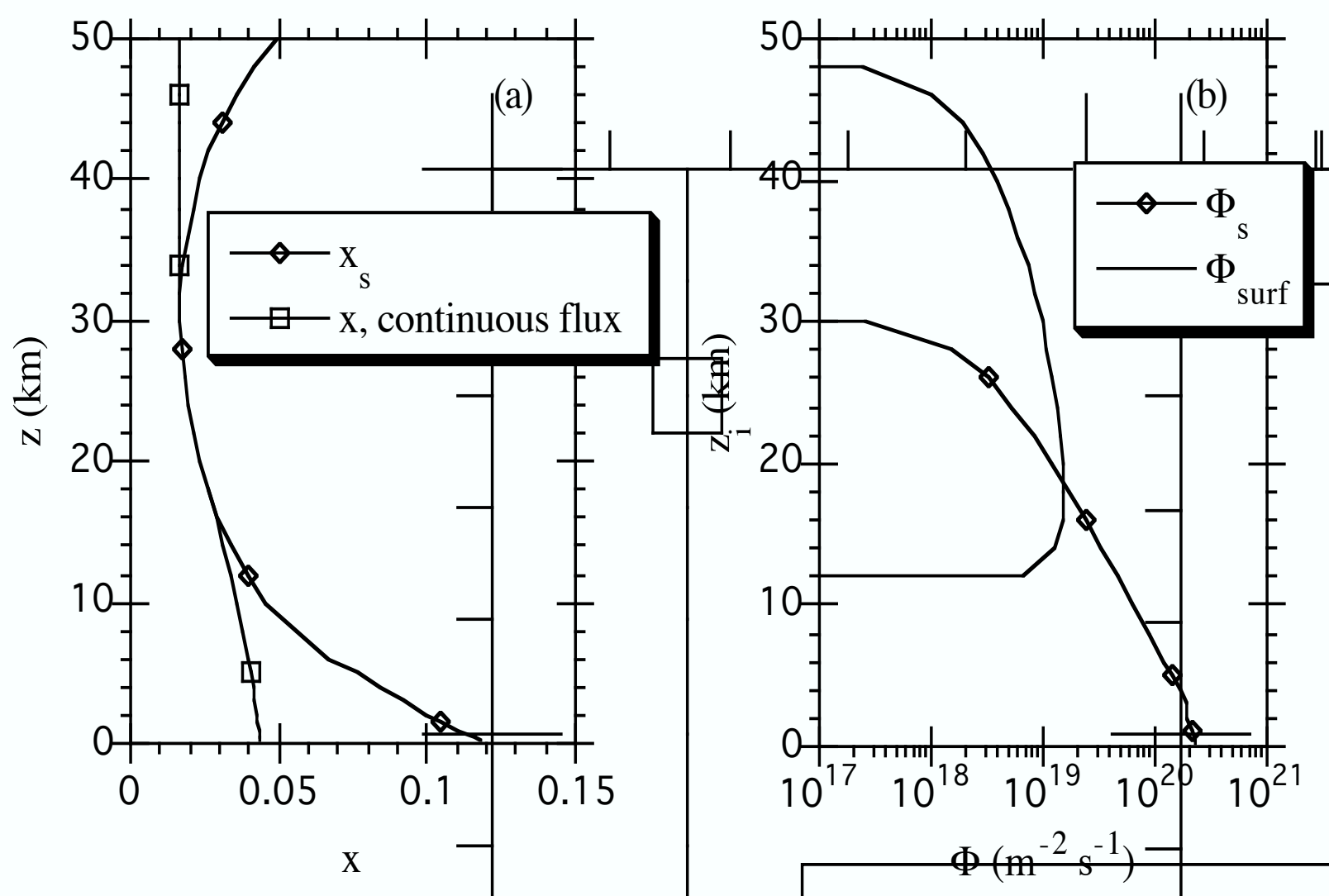

Figure 1 


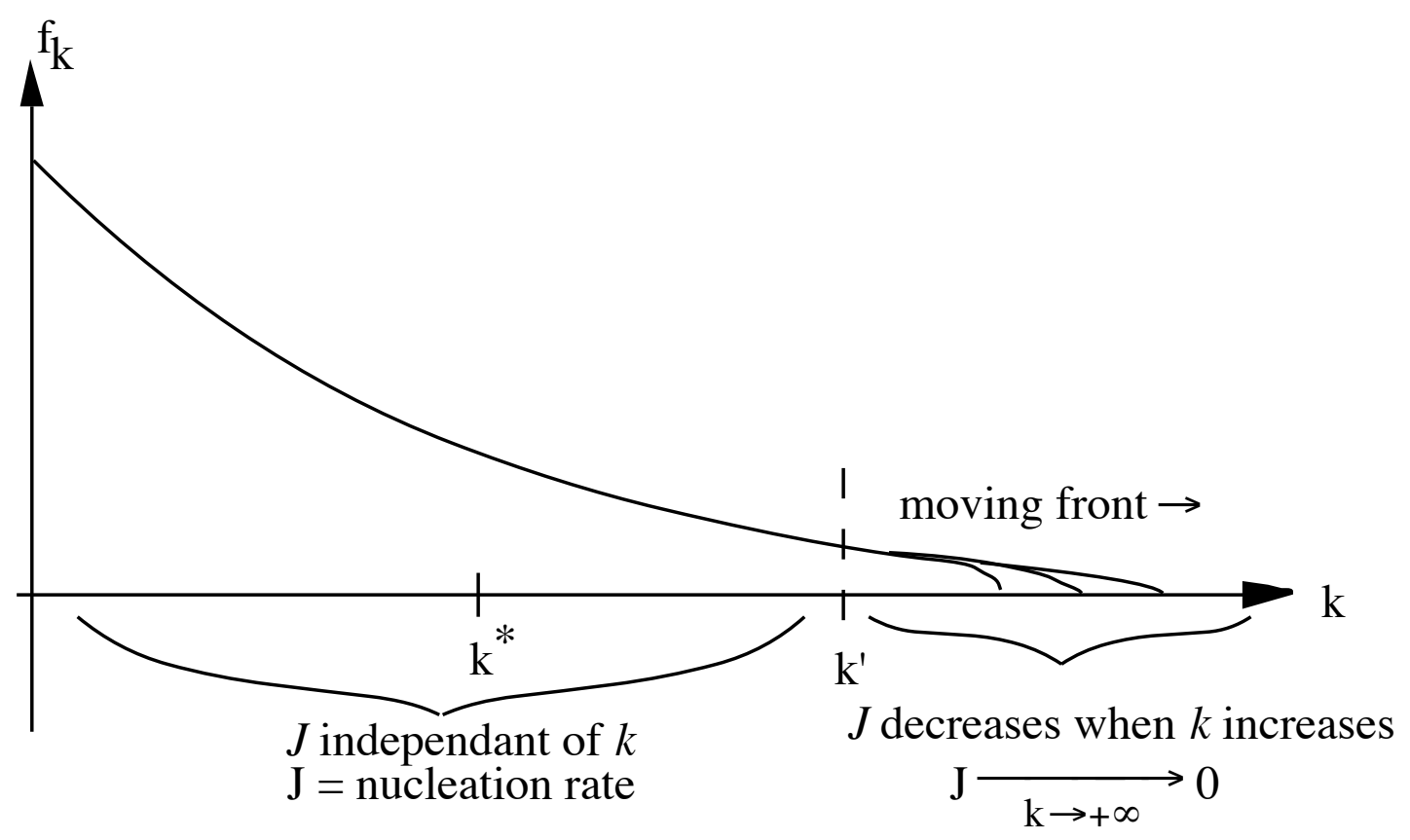

Figure 2 


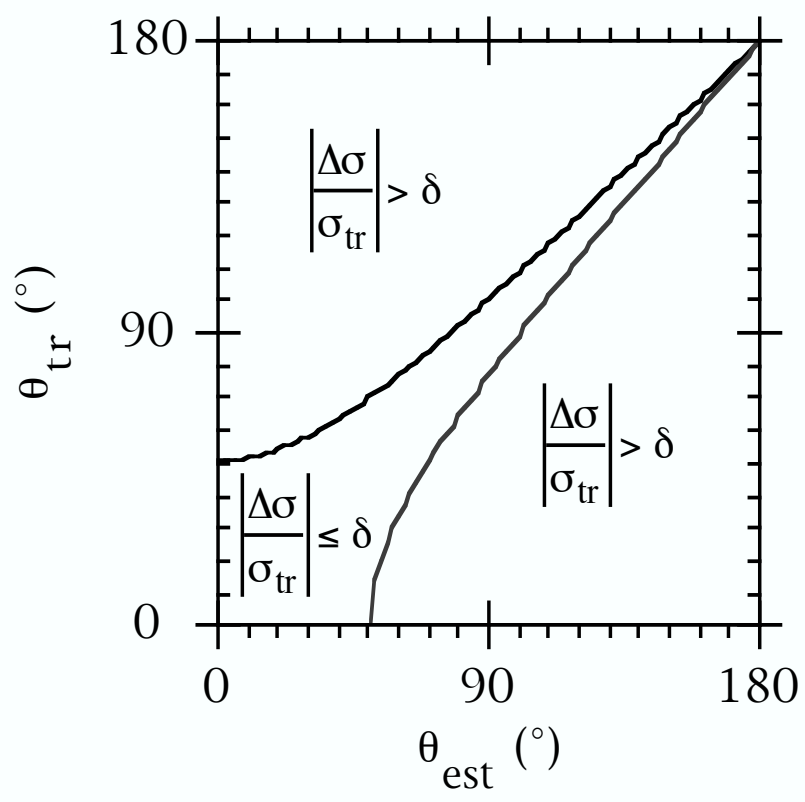

Figure 3 


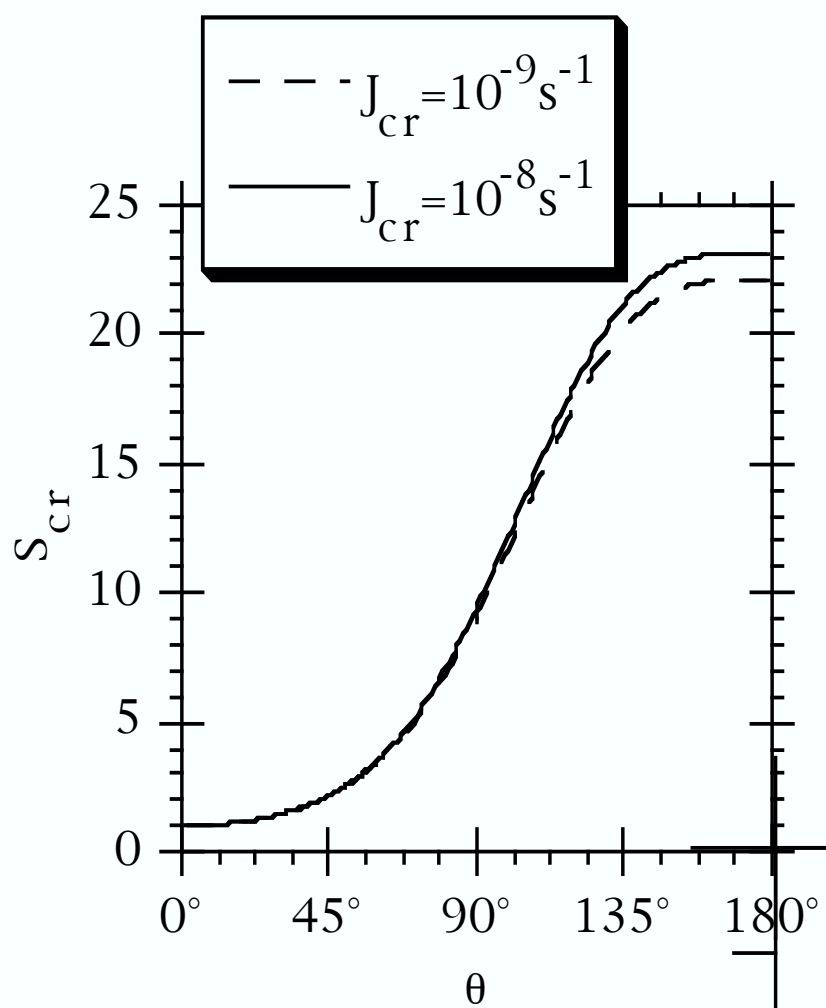

Figure 4 


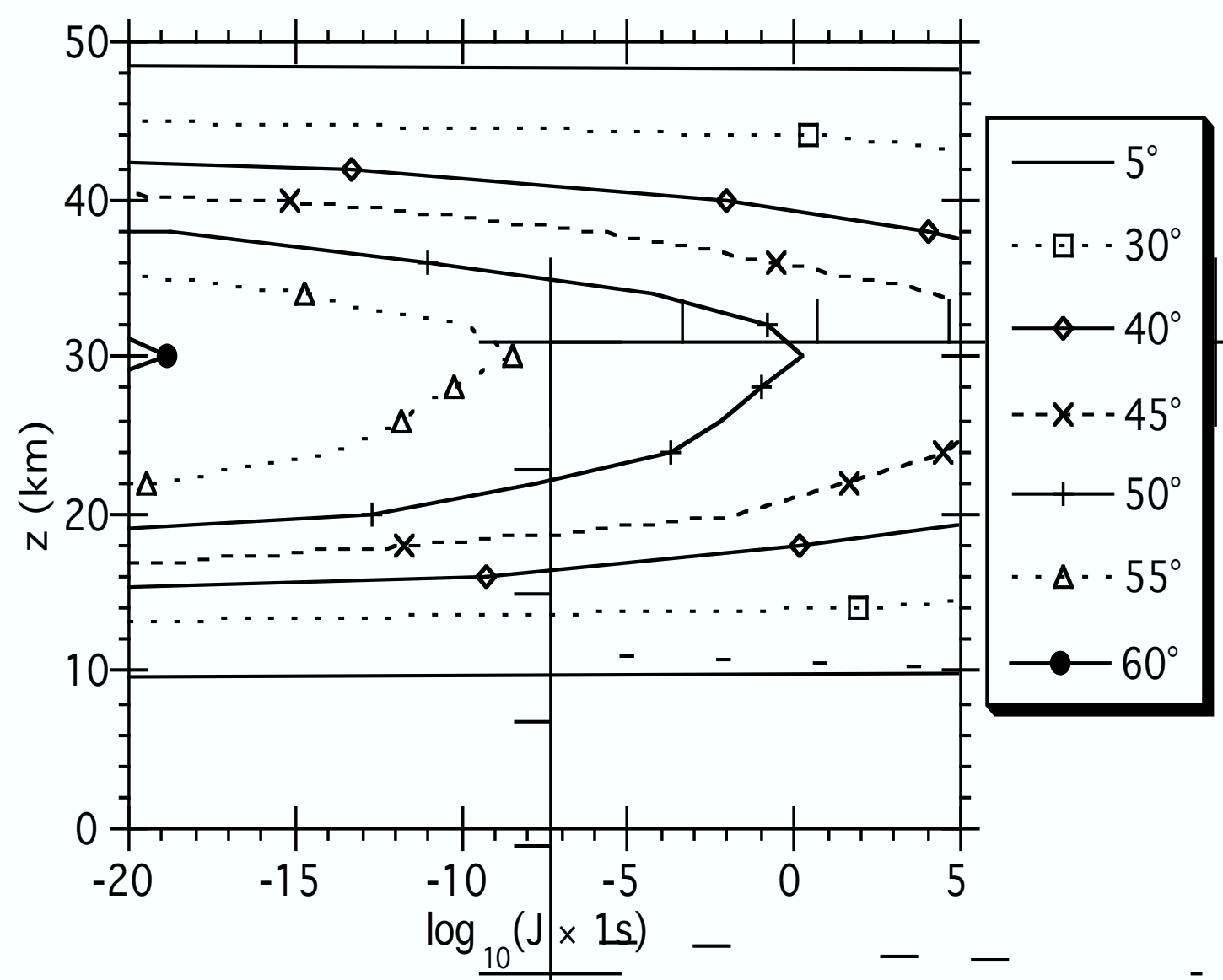

Figure 5 


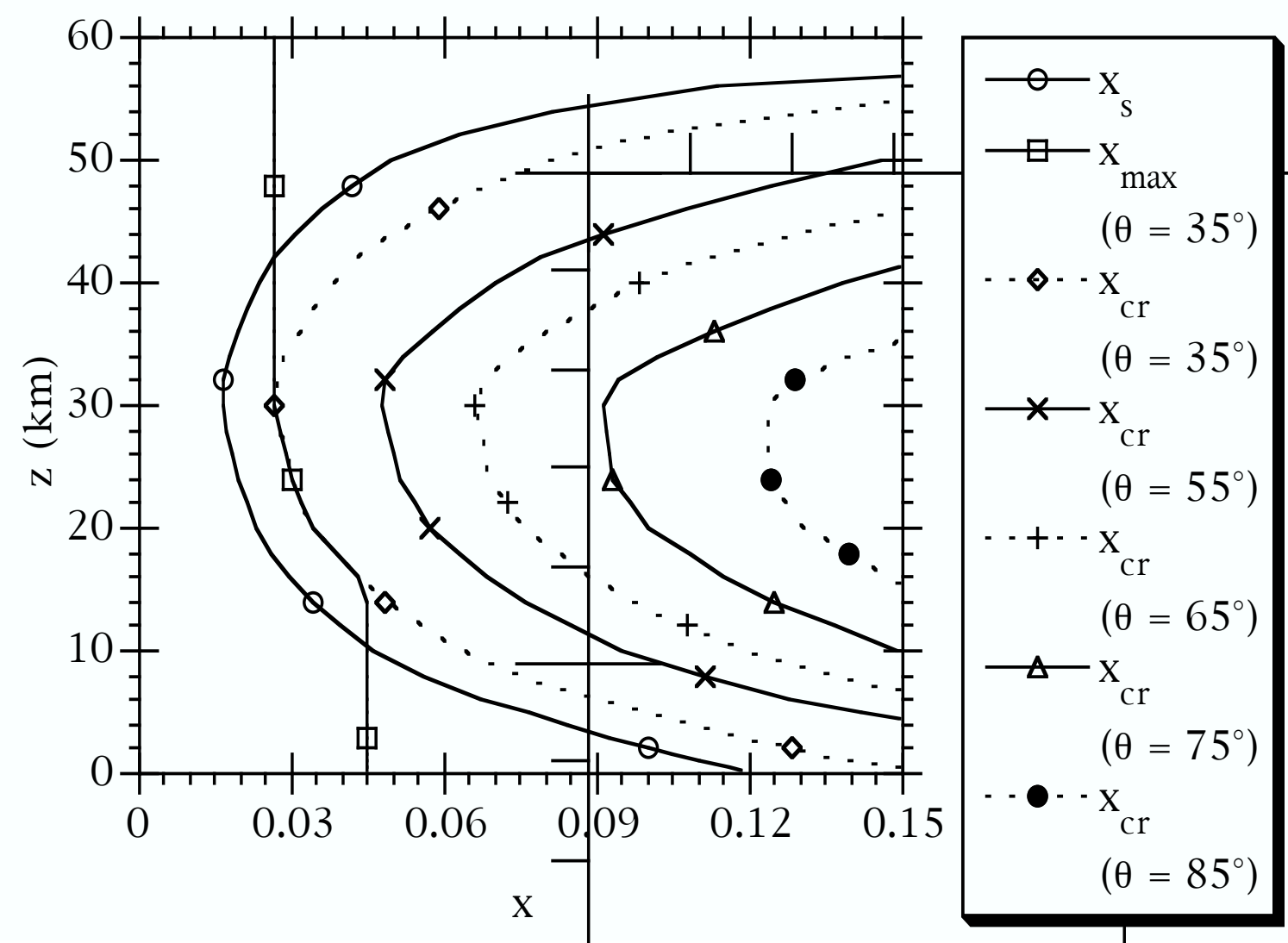

Figure 6 


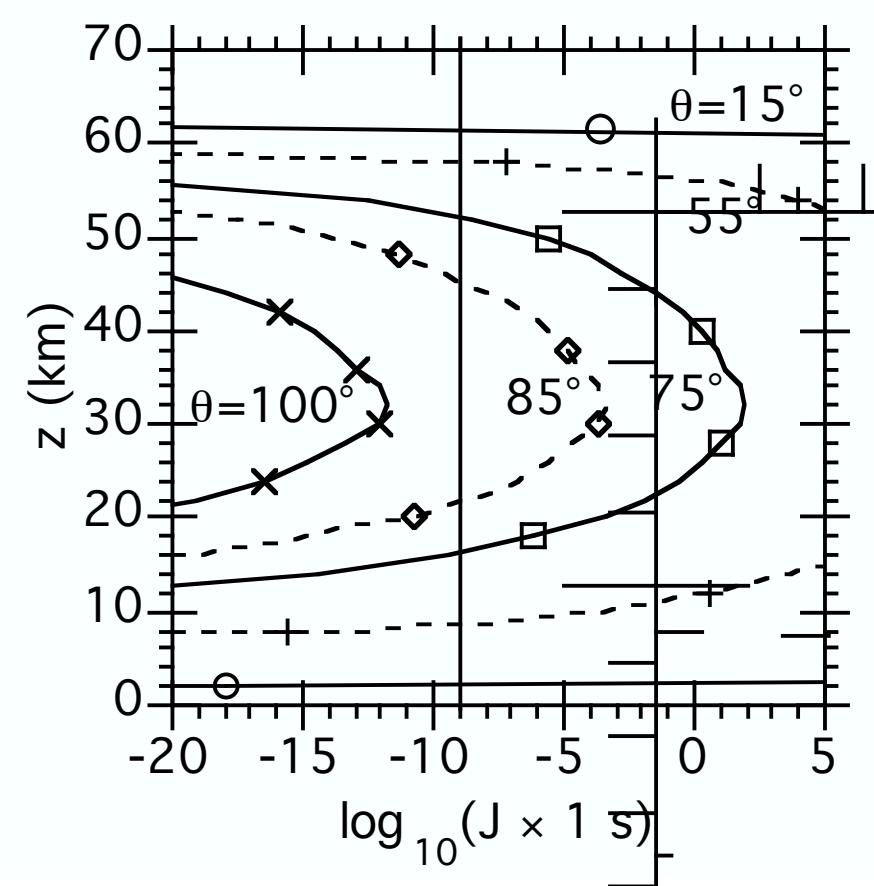

Figure 7 